\title{
Vortex Physics in Confined Geometries
}

\author{
M. Cristina Marchetti * and David R. Nelson \\ Lyman Laboratory of Physics, Harvard University, Cambridge, MA 01238
}

(May 13, 2017)

\begin{abstract}
PACS: $74.60 \mathrm{Ge}$
Keywords: Vortex arrays, hydrodynamics, Bose glass, scaling.

Patterned irradiation of cuprate superconductors with columnar defects allows a new generation of experiments which can probe the properties of vortex liquids by forcing them to flow in confined geometries. Such experiments can be used to distinguish experimentally between continuous disorder-driven glass transitions of vortex matter, such as the vortex glass or the Bose glass transition, and nonequilibrium polymer-like glass transitions driven by interaction and entanglement. For continuous glass transitions, an analysis of such experiments that combines an inhomogeneous scaling theory with the hydrodynamic description of viscous flow of vortex liquids can be used to infer the critical behavior. After generalizing vortex hydrodynamics to incorporate currents and field gradients both longitudinal and transverse to the applied field, the critical exponents for all six vortex liquid viscosities are obtained. In particular, the shear viscosity is predicted to diverge as $\left|T-T_{B G}\right|^{-\nu z}$ at the Bose glass transition, with $\nu \simeq 1$ and $z \simeq 4.6$ the dynamical critical exponent. The scaling behavior of the ac resistivity is also derived. As concrete examples of flux flow in confined geometries, flow in a channel and in the Corbino disk geometry are discussed in detail. Finally, the implications of scaling for the hydrodynamic description of transport in the dc flux transformer geometry are discussed.
\end{abstract}

\section{INTRODUCTION}

In the mixed state of cuprate superconductors the magnetic field is concentratedinan array of flexible flux bundles that, much like ordinary matter, can form crystalline, liquid and glassy phases.t 1 B The dynamics of the flux-line array determines the resistive properties of the material and has therefore been the focus of much theoretical and experimental work. Of particular interest are the phase transitions connecting various forms of vortex matter. For example, a first order melting transition is now believed to connect the crystalline Abrikosov flux lattice to a melted flux liquid. If the barriers to line crossings are sufficiently high rapidly cooled vortex liquids can bypass the solid

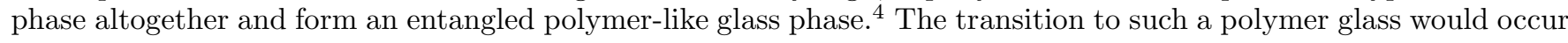
when the system gets trapped in a metastable state, much like the transition in ordinary window glass.

Other types of glasses are also possible because of pinning in disordered samples. It was suggested some time ago that point disorder may driife a continuous transition from a vortex liquid to an isotropic vortex glass state, with vanishing linear resistivity. 0 6 The existence of this transition is still in doubt, as many of the early experiments claiming to observe it $\mathbf{3}$ were in fact dominated by twin boundary pinning. The vortex glass remains, however, a credible candidate for the description of the disordered solid phase observed in untwinned crystals at high fields.

Correlated disorder, that is disorder that can pin vortex lines coherently along a specific direction, is also very important in many materials. It can be created artificially via the introduction of columnar damage tracks created by heavyion irradiation - a procedure that was shown to result in dramatic improvement in the pinning of vortex lines.10 12 It can alspbe present in the material in the form of families of parallel twin planes that pass completely through the sample.13 In samples with columnar disorder, if the concentration of damage tracks (assumed to pass completely through the sample) exceeds the number of flux lines, there is a low-temperature "Bose glass" phase, in which every vortex is trapped on a columnar defect and the linear resistivity vanishes. 14 At high temperatures the vortices delocalize in an entangled flux-line liquid. The high temperature liquid transforms into a Bose glass

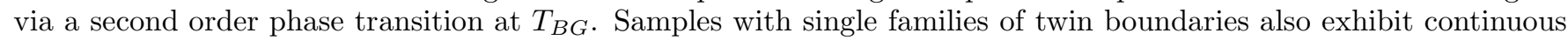
transitions to anisotropic glass phases.15.16

\footnotetext{
${ }^{*}$ Corresponding author. Permanent address: Physics Department, Syracuse University, Syracuse, NY 13244; Tel. (315)4432581; Fax (315)443-9103; email:mcm@physics.syr.edu
} 
The Bose glass transition is the one that is best understood and experimentally characterized. For this reason we will use it in much of the following as our prototype of a disorder-driven continuous glass transition in vortex matter. It has been studied theoretically by viewing the vortex line trajectories as the world lines of two-dimensional quantum mechanical particlest 14 . The thickness of the superconducting sample corresponds to the inverse temperature of the quantum particles. In thick samples the physics of vortex lines pinned by columnar defects becomes equivalent to the low temperature properties of two-dimensional bosons with point disorder. In the low temperature phase Bose glass phase the vortices behave like localized bosons. The entangled flux liquid phase is resistive and corresponds to a boson superfluid. For simplicity, we confine our attention here to fields less that the "matching field" $B_{\phi}=n_{\text {pin }} \phi_{0}$, with $n_{\text {pin }}$ the areal density of columnar pins and $\phi_{0}=\hbar c / 2 e$ the flux quantum.

Although an exact theory of such continuous transitions from the Bose glass or from the vortex glass to the entangled flux liquid phase is not available, near the transition most physigal properties can be described via a scaling theory in terms of just two undetermined critical exponent, 6, 14, 17, $18,20,21$. The low temperature disorder-dominated glass phase is characterized by a a correlation volume whose size diverges at the transition. Anisotropic disorder, such as columnar defects or twin planes, results in an anisotropic correlation volume. The most complicated case is that of planar disorder, embodied for instance by a single family of parallel twin planes. In this case there are three correlation lengths that diverge at the continuous glass transition temperature, $T_{G}$ (see Fig. 1). The growth of correlations in the direction perpendicular to both the external field and the twin planes is described by the correlation length

$$
\xi_{\perp}(T) \sim\left|T-T_{G}\right|^{-\nu} .
$$

A second diverging length, $\tilde{\xi}_{\perp}(T)$, describes the extent of correlations in the direction perpendicular to the external field, but parallel to the plane of the twin boundaries,

$$
\tilde{\xi}_{\perp}(T) \sim\left|T-T_{G}\right|^{-\tilde{\nu}}
$$

Finally, there is a diverging correlation length along the external field (here the $z$ direction), which is aligned with the twin planes,

$$
\xi_{\|}(T) \sim \xi_{\perp}^{\zeta} \sim\left|T-T_{G}\right|^{-\nu \zeta}
$$

with $\zeta$ the anisotropy exponent. For planar disorder $\zeta=1+\tilde{\nu} / \nu$ (this constraint follows from the finiteness of $c_{11}$ at the transition). In materials with columnar pins aligned with the external field the correlation volume is isotropic in the plane normal to the columns. Thus $\xi_{\perp}=\tilde{\xi}_{\perp}$ and there are two correlation lengths that diverge at the Bose glass transition transition temperature, $T_{B G}$, characterizing correlations in the directions perpendicular $\left(\xi_{\perp}\right)$ and parallel $\left(\xi_{\|}\right)$to the linear defects. In this case the anisotropy exponent $\zeta$ has the value $\zeta=2$. The same results are expected to hold for a mosaic of twin boundary planes, all containing the field direction, on scales large compared to the mosaic size. Finally, for the case of point disorder controlling the transition to the vortex glass phase at $T_{V G}$, the correlation volume scaling is assumed to be isotropic. 6 There is only a single diverging correlation length, $\xi_{\perp}$, with $\zeta=1$, although it is hard to rule out different length scales parallel and perpendicular to the field direction. The correlation time controlling the relaxation of critical fluctuations is assumed to diverge as

$$
\tau \sim \xi_{\perp}^{z} \sim\left|T-T_{G}\right|^{-z \nu}
$$

with $z$ the dynamical critical exponent. The values of the critical exponents $\nu$ and $z$ of course depend on the type of disorder and differ for instance for the Bose glass and the vortex glass For the Bose glass transition the critical exponents have been determined via simulations to be $\nu \simeq 1$ and $z \simeq 4.6 \mathrm{e}$.

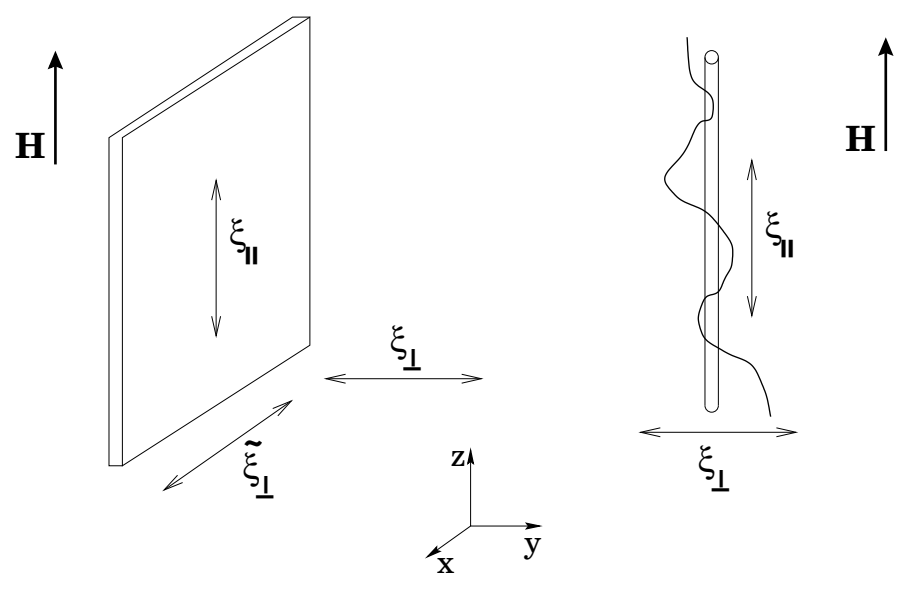


Figure 1. The two anisotropic disorder geometries discussed in the text and the corresponding diverging correlation lengths.

Scaling can then be used to relate physical quantities to these diverging correlation lengths and time. In particular, the linear resistivity $\rho_{\perp}(T)$ of the vortex liquid for currents applied in the $a b$ plane is predicted to vanish as $T \rightarrow T_{G}$ from above as

$$
\rho_{\perp}(T) \sim\left|T-T_{G}\right|^{\nu(z-\zeta)}
$$

in bulk samples in three dimensions 14.

Although some predictions of the scaling theory have been tested experimentally, there are as yet no direct measurements of the transport coefficients usually associated with glass transitions in conventional forms of matter, such as the shear viscosity. In this paper we show that the liquid viscosities also exhibit strong divergences at a continuous disorder-driven glass transition of vortex matter. We make explicit predictions for the critical exponents controlling the divergence of the liquid viscosities and propose experiments which test our predictions. For instance, the behavior of the shear viscosity is found to be determined by the dynamical critical exponent $z$ that controls the divergence of the relaxation time in the glass phase. A direct measurement of the shear viscosity would therefore provide a direct probe of the diyerging relaxation time associated with glassy behavior.22 A brief account of this work has been presented elsewhere.23

As for ordinary matter, the shear rigidity of the vortex array can be probed by driving the vortices to flowin confined geometries 24 The fabrication and use of such confined geometries was pioneered by Kes and collaborators 252 to study the shear rigidity of the two-dimensional vortex liquid near the freezing transition in thin superconducting films. More recently, patterned irradiation of cuprate superconductors with heavy ions has made it possible to create samples with controlled distributions of damage tracks that will allow for a new generation of experiments that may in fact provide a direct probe of viscous critical behavior near the Bose glass transition 26 . Specifically, by starting with a clean sample, at temperatures such that point disorder is negligible, it is possible to selectively irradiate regions of controlled geometry by covering the top of the sample with a suitable mask. Perhaps the simplest geometry that can be created is that of the channel shown in Fig. 2. This closely parallels an experimental setup proposed several years ago for measwring the shear viscosity of an entangled vortex liquid forced to flow in the channel between two parallel twin planes.24 Now we propose that a similar geometry created by selective heavy ion irradiation may be used to probe the growing correlations at the Bose glass transition.23

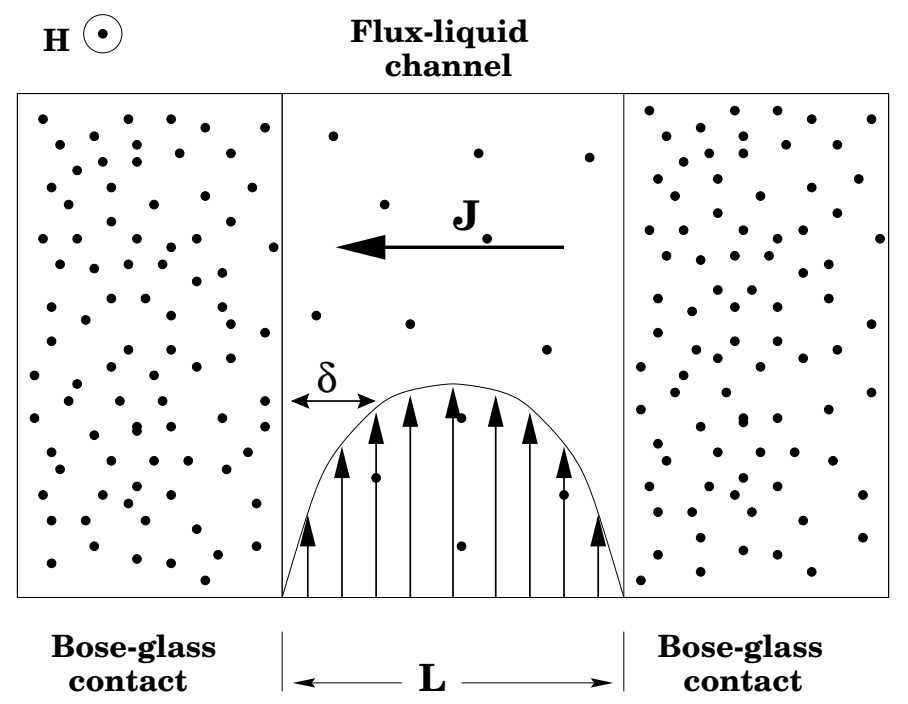

Figure 2. A weakly irradiated channel where the flux liquid is sandwiched between two heavily irradiated Boseglass contacts. A current $J$ applied across the channel yields flux motion along the channel. Vortex pinning at the boundaries propagates a length $\delta$ into the channel, yielding a spatially inhomogeneous flow profile. 
As shown in Fig. 2, the side regions of the samples have been heavily irradiated, and are characterized by a high matching field $B_{\phi}^{(2)}$ and transition curve $T_{B G}^{(2)}$, while the channel is lightly irradiated with a lower matching field $B_{\phi}^{(1)}<B_{\phi}^{(2)}$ and transition curve $T_{B G}^{(1)}$. When $T_{B G}^{(1)}<T_{B G}<T_{B G}^{(2)}$, the flux array in the channel is in the liquid state, while the contacts are in the Bose glass phase. Flow in the resistive flux liquid region is impeded by the "Boseglass contacts" at the boundaries, as the many trapped vortices in these regions provide an essentially impenetrable barrier for the flowing vortices, due to their mutual interactions. As the temperature is lowered at constant field, so that the Bose glass transition $T_{B G}^{(1)}$ of the liquid region is approached from above (Fig. 3) the Bose glass correlation length increases, forcing the pinning at the boundaries to propagate into the liquid channel and yielding a spatially inhomogeneous electric field profile which can be probed experimentally. We will show that the spatial inhomogeneity of the electric field occurs precisely on length scales given by the correlation length, providing an unambiguous prescription for extracting critical exponents from this type of transport measurements.

By controlling the disorder in the channel region, the same geometry can also be used to study the onset of rigidity in the vortex array near the vortex glass or the polymer glass transitions. In the former case the channel (now free $\rho$ damage tracks) should contain a high concentration of point defects (augmented, perhaps, by proton irradiation 30). The polymer glass transition may take place when the channel is "clean", with only a low concentration of oxygen vacancies. A crucial difference between the polymer glass transition and the continuous disorder-driven glass transitions discussed above is that the former is a nonequilibrium phenomenon associated with the slowing down of the system dynamics on experimental time scales and does not exhibit universal critical behavior. For this reason, by testing for a divergent length scale, transport measurements in confined geometries may actually be used to distinguish experimentally between a continuous disorder-driven glass transition and the polymer glass transition.27

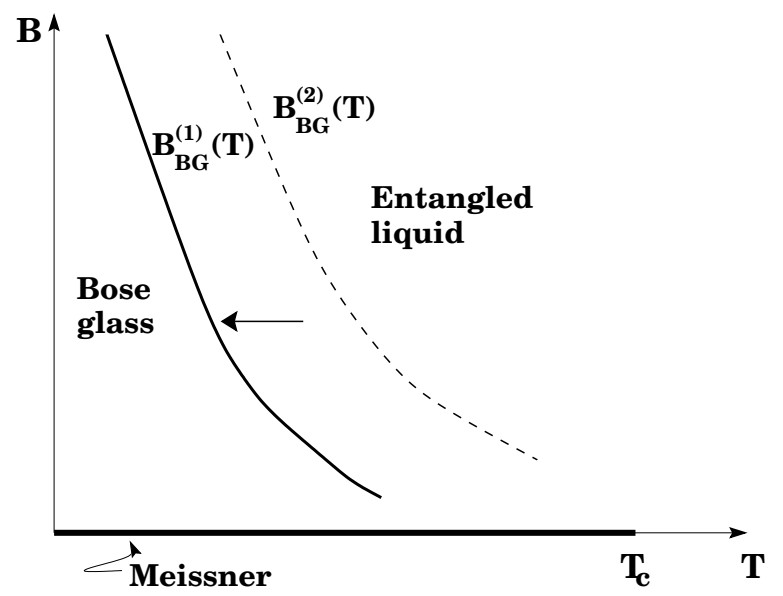

Figure 3. A sketch of the $(B, T)$ phase diagram for the flux array in the weakly irradiated channel region. The heavy line, $B_{B G}^{(1)}(T)$, denotes the continuous transition from the Bose glass to the entangled liquid. Also shown is the location $B_{B G}^{(2)}(T)$ of the Bose glass transition line in the heavily irradiated contacts. When a field $B_{B G}^{(1)}(T)<B<B_{B G}^{(2)}(T)$ is applied, the flux array in the channel is in the liquid state, while the contacts are in the Bose glass phase. By decreasing the temperature at constants field along the direction of the arrow, the Bose glass transition of the channel region is approached from above. When $\bar{B}$, instead of $H$, is used for the phase diagram, the Meissner phase collapses to a line, as shown.

As shown in Ref. 24, the electrodynamics of vortex liquids is in general nonlocal due to interactions and entanglement, even away from phase transitions. Such nonlocalities may become very important near a phase transition, particularly when the transition is continuous. A natural way of incorporating the nonlocality in the long wavelength description of vortex liquid dynamics is via a set of hydrodynamic equations where the effect of intervortex interactions appears as viscous forces. A complete set of hydrodynamic equations for the vortex liquid is given in section II. These equations incorporate driving forces from external current both parallel and transverse to the field $\mathbf{H}$, as well as both compressional forces and pressure forces due to vortex segments that are not aligned with the $z$ axis. They therefore generalize earlier hydrodynamic equations written down by us that only considered flow driven by currents in the $a b$ plane 24 As pointed out earlier by Mou et al 28, the line nature of vortex matter yields to a proliferation 
of viscosity coefficients, much like in liquid crystals. There are therefore six independent vortex liquid viscosities in these equations. The general equations are complicated and will be discussed in more detail below. The equation for the coarse-grained flow velocity $\mathbf{v}(\mathbf{r})$ of the vortex liquid in the channel geometry of Fig. 2 is, however, very simple, namely 24

$$
-\gamma_{\perp} \mathbf{v}+\eta \nabla_{\perp}^{2} \mathbf{v}=\frac{1}{c} n_{0} \phi_{0} \hat{\mathbf{z}} \times \mathbf{J}
$$

where $\gamma_{\perp}$ is the friction coefficient that incorporates both the interaction of the vortex cores with the underlying ionic lattice and the pinning by material defects and $\eta$ is the vortex liquid shear viscosity. The term on the right hand side of Eq. (1.6) is the Lorentz current due to an external current $\mathbf{J}$ applied across the channel, with $n_{0}$ the areal density of vortices. Since the local electric field from flux motion is $\mathbf{e}(\mathbf{r})=\frac{n_{0} \phi_{0}}{c} \hat{z} \times \mathbf{v}(\mathbf{r})$, Eq. (1.6) can be rewritten as a nonlocal version of Ohm's law,

$$
\mathbf{e}-\delta^{2} \nabla_{\perp}^{2} \mathbf{e}=\rho_{\perp} \mathbf{J}
$$

where

$$
\rho_{\perp}(T)=\left(\frac{n_{0} \phi_{0}}{c}\right)^{2} \frac{1}{\gamma_{\perp}(T)}
$$

is the in-plane linear resistivity in a bulk sample. The viscous length

$$
\delta(T)=\sqrt{\eta(T) / \gamma_{\perp}(T)}
$$

controls the importance of viscous drag. In a bulk sample and in the absence of strong inhomogeneities in the spatial distribution of disorder, the electrodynamic response is expected to be essentially local on large scales. Far from the boundaries in macroscopic samples, the viscous drag drops out from Eq. (1.7) and the linear vortex liquid resistivity is given by Eq. (1.8) and is controlled entirely by the friction coefficient, $\gamma_{\perp}$. In contrast, in a confined geometry viscous drag becomes dominant near a glass transition and the viscous length controls the flux liquid resistivity in this case.

For continuous disorder-driven glass transitions, such as the vortex or Bose glass transitions, a dimensional analysis of the hydrodynamic equations, combined with the scaling hypothesis that the diverging correlation lengths $\xi_{\perp}$ and $\xi_{\|}$are the only relevant length scales in the problem, leads to the identification of the viscous length with the in-plane correlation length,

$$
\delta(T) \sim \xi_{\perp}(T) \sim\left|T-T_{G}\right|^{-\nu} .
$$

Since the bulk resistivity vanishes at $T_{B G}$ according to Eq. (1.5), or equivalently the friction $\gamma_{\perp}(T)$ diverges with the same exponent,

$$
\gamma_{\perp}(T) \sim\left|T-T_{B G}\right|^{-\nu(z-\zeta)},
$$

the identification of these two length scales immediately leads to the prediction that the liquid shear viscosity diverges at a continuous glass transition as

$$
\eta(T) \sim \xi_{\perp}^{2}(T) \gamma_{\perp}(T) \sim\left|T-T_{B G}\right|^{-\nu(z+2-\zeta)} .
$$

At the Bose glass transition, where $\zeta=2$ and $\nu \simeq 1$, the divergence of the shear viscosity is controlled by the dynamical exponent $z$.

In contrast, there is no obvious diverging correlation length controlling the polymer glass transition proposed some time ago by one of us $t$ as an alternative to the vortex glass scenario and a possible explanation for the irreversibility line observed experimentally at high fields. In this scenario a viscous vortex liquid with large barriers to line crossing, when cooled rapidly may drop out of equilibrium at a polymer glass transition temperature $T_{P G}$, well before it has time to nucleate the equilibrium crystalline phase. The polymer glass is metastable and the transition is analogous to the glass transition conjectured in ordinary forms of matter. Disorder plays no role in the transition itself (although it may have dramatic effects in controlling the dynamical response of the vortex array) and the vortex friction $\gamma_{\perp}$ remains finite across the transition. The shear viscosity grows rapidly as $T \rightarrow T_{P G}^{+}$and its temperature dependence might conceivably be described by the standard Vogel-Fulcher form,

$$
\eta(T) \approx \eta_{0} e^{c /\left(T-T_{P G}\right)},
$$


which works very well for glass transitions in conventional forms of matter. Experiments capable of measuring both the friction coefficient $\gamma_{\perp}$ (which can be extracted from a bulk resistive measurements) and the viscous length $\delta$ (which can be obtained from resistive measurements in confined geometries) can therefore distinguish between a kinetic polymer glass transition and a continuous-disgrder driven glass transition, as well as providing direct measurements of the critical exponents in the latter case.27 At a continuous glass transition, both friction and viscosity will diverge with a characteristic critical exponent. At a polymer glass transition, the friction will remain finite and the viscosity is expected to exhibit the Vogel-Fulcher behavior given by Eq. (1.13).

As shown in section III, dimensional analysis of the general hydrodynamic equations yields predictions for the scaling exponents for all the six vortex liquid viscosities near a continuous glass transition. The results (see Sec. II for the precise definition of these quantities) are summarized in table 1.

\begin{tabular}{|c|c|}
\hline$\gamma_{\perp}$ & $\nu(z-\zeta)$ \\
\hline$\gamma_{\Perp}$ & $\nu(z-2+\zeta)$ \\
\hline$\eta$ & $\nu(z+2-\zeta)$ \\
\hline$\eta_{b}$ & $\nu(z+2-\zeta)$ \\
\hline$\eta_{z}$ & $\nu(z+\zeta)$ \\
\hline$\eta^{t}$ & $\nu(z+\zeta)$ \\
\hline$\eta_{z}^{t}$ & $\nu(z-2+3 \zeta)$ \\
\hline$\eta_{x}$ & $\nu(z+\zeta)$ \\
\hline
\end{tabular}

Table 1. A summary of the critical exponents for the friction and viscosity coefficients (see Sec. II for definitions) near a continuous glass transition in three dimensions. Each transport coefficient diverges at $T_{B G}$ as $\sim t^{-\alpha}$, where $t=\left|T-T_{B G}\right| / T_{B G}$. The value of $\alpha$ for each transport coefficient is indicated in the second column. The anisotropy exponent $\zeta$ should be chosen as $\zeta=2$ for the Bose glass transition and $\zeta=1$ for the vortex glass transition.

We stress that the identification of the dynamical length scale $\delta$ with the static correlation length $\xi_{\perp}$ is by no means obvious. It mirrors the corresponding result obtained in the theory of continuous melting in two dimensions, where the liquid shear viscosity scales as $\eta \sim 1 / \xi_{d}^{2}$, with $\xi_{d}$ the average distance between unbound dislocations, which in turn diverges at the transition, yielding a corresponding divergence of the viscosity 29 In this case the precise relationship between static and dynamical properties is one of the outcomes of the theory of two-dimensional melting which occurs via successive dislocation and disclination unbinding. For the vortex or Bose glass transitions the divergence of the correlation length is due to collective effects mediated by extrinsic quenched disorder. The assumption made here is that this growing correlations associated with disorder will also drive the divergence of dynamical properties in the liquid phase, namely the viscosities.

This hypothesis has powerful implications. As shown in Ref. 23 and described in more detail in section IV below, by combining scaling in spatially inhomogeneous geometries with hydrodynamics we are able to obtain not just the scaling exponents for the transport coefficients, but also the full form of the scaling function for the linear resistivity of the vortex liquid in a constrained geometry. The precise form of the scaling function depends on the details of the geemetry and was given in 23 for the channel and for the Corbino disk geometry (Fig. 4) used recently by López et al.30. Once the form of the scaling function is known, a quantitative analysis of transport experiments can in principle be carried out to extract the critical exponents.

The plan of the paper is as follows. In section II we generalize the hydrodynamic equations of Ref. 24 to incorporate gradients and driving forces in both the directions parallel and transverse to the applied field. Upon neglecting the small transverse frictional forces responsible for the Hall effect, the hydrodynamic equations contain two friction coefficients and six viscosity coefficients. In section [II], after briefly summarizing the scaling theory for the Bose glass transition in bulk, we discuss the dimensional analysis of the hydrodynamic equations and obtain the critical exponents for all the transport coefficients, as given in table 1. In section [V] we combine scaling in spatially inhomogeneous systems with hydrodynamics to show how the full form of the scaling function can be obtained for specific geometries. The Corbino disk geometry is discussed in some detail. Finally, section V generalizes both the scaling theory and the hydrodynamics to finite frequency to discuss the critical scaling of the linear response of the vertex liquid to ac drives at the Bose glass transition. Appendix A makes contact with the earlier work by Mou et al.28 on nonlocal effects in vortex liquids. In appendix B we apply scaling ideas to the analysis of transport in the dc flux transformer geometry. 


\section{HYDRODYNAMICS OF FLUX-LINE LIQUIDS}

Here we generalize the hydrodynamics of flux-line liquids discussed earlier by us 24 to incorporate the effect of driving currents in arbitrary directions, with components both parallel and perpendicular to the external field $\mathbf{H}_{0}=\hat{\mathbf{z}} H_{0}$ responsible for the onset $\mathrm{gf}$ the vortex state. The resulting hydrodynamic equations contain the six viscosity coefficients discussed by Mou et al 28 that control the nonlocal electrodynamic response of flux liquids.

The electrodynamics of a type-II superconductor in the mixed state is described by Maxwell's equations for the local fields $\mathbf{b}(\mathbf{r}, t)$ and $\mathbf{e}(\mathbf{r}, t)$,

$$
\begin{aligned}
& \frac{1}{c} \partial_{t} \mathbf{b}+\nabla \times \mathbf{e}=0, \\
& \nabla \times \mathbf{b}=\frac{4 \pi}{c} \mathbf{j},
\end{aligned}
$$

where we have dropped the displacement current which is negligible at all but very high frequencies $\left(\omega \simeq c / \lambda \sim 10^{15} \mathrm{~Hz}\right)$ and $\mathbf{j}$ denotes the total current density, including the equilibrium response of the medium, in addition to any external current. In addition, the fields satisfy divergence equations,

$$
\begin{aligned}
& \boldsymbol{\nabla} \cdot \mathbf{b}=0, \\
& \boldsymbol{\nabla} \cdot \mathbf{e}=4 \pi \rho .
\end{aligned}
$$

Both fields can be obtained from a vector potential $\mathbf{A}$ and a scalar potential $\phi$, according to $\mathbf{b}=\boldsymbol{\nabla} \times \mathbf{A}$ and $\mathbf{e}=-\frac{1}{c} \partial_{t} \mathbf{A}-\boldsymbol{\nabla} \phi$. We work here with the Coulomb gauge, $\boldsymbol{\nabla} \cdot \mathbf{A}=0$. The equations for the fields must be supplemented with a constitutive equation for the current $\mathbf{j}$. In the linear (Ohmic) regime this is simply given by a nonlocal generalization of Ohm's law,

$$
j_{\alpha}(\mathbf{r})=\int_{\mathbf{r}^{\prime}} \Sigma_{\alpha \beta}\left(\mathbf{r}, \mathbf{r}^{\prime}\right) e_{\beta}\left(\mathbf{r}^{\prime}\right),
$$

where Greek indices $\alpha, \beta, \ldots$ run over the values $x, y, z$ and are used to denote Cartesian components in three dimensions and $\Sigma_{\alpha \beta}$ is a nonlocal conductivity tensor. Below we will also use Latin indices $i, j, \ldots$ to denote Cartesian coordinates in the $x y$ plane, i.e., Latin indices will only assume values $x$ and $y$. Nonlocality must be incorporated when describing the mixed state of the cuprates where interactions and entanglement among the vortices can yield long-ranged correlations and large scale nonlocal electrodynamic response. It is useful for the following to rewrite Eq. (2.5) by separating the current in an external and internal contributions, $\mathbf{j}=\mathbf{J}+\mathbf{j}^{\text {int }}$, with $\mathbf{j}^{\text {int }}=\frac{c}{4 \pi} \boldsymbol{\nabla} \times(\mathbf{b}-\mathbf{H})$. Equation (2.5) can then be written as

$$
J_{\alpha}(\mathbf{r})-c\left(\boldsymbol{\nabla} \times \frac{\delta F}{\delta \mathbf{b}}\right)_{\alpha}=\int_{\mathbf{r}^{\prime}} \Sigma_{\alpha \beta}\left(\mathbf{r}, \mathbf{r}^{\prime}\right) e_{\beta}\left(\mathbf{r}^{\prime}\right),
$$

where $-4 \pi \frac{\delta F}{\delta \mathbf{b}}=\mathbf{b}-\mathbf{H}$ represents the local magnetization of the medium, with $F(T, \mathbf{b})$ the Helmoltz free energy of the superconductor in an external field, H. The second term on the left hand side of Eq. 2.6) incorporates the static Meissner response of the material as well as the current due to local Magnus and pressure forces from the vortices.

In this paper we are interested in fluctuations in the $\mathbf{b}$ and $\mathbf{e}$ fields due to fluctuations in the vortices degrees of freedom. The relationship between the local supercurrent, $\mathbf{j}_{s}$, and the vortex degrees of freedom is obtained by minimizing the Ginzburg-Landau free energy functional for fixed vortex configurations. Note that in general by using a two-fluid picture there will also be a "normal current" contribution to the internal current, $\mathbf{j}^{\text {int }}=\mathbf{j}_{s}+\mathbf{j}_{n}$. We will not incorporate this normal part of the response here. In the London approximation, where the magnitude of the superconducting order parameter is assumed constant, and only fluctuations in its phase, $\theta$, are retained, one obtains the usual London equation,

$$
\lambda^{2} \mathbf{j}_{s}=-\frac{c}{4 \pi}\left(\mathbf{A}-\phi_{0} \nabla \theta\right)
$$

where $\lambda$ is the penetration length and $\phi_{0}=h c / 2 e$ the flux quantum. For clarity here we discuss only the case of an isotropic superconductor. The equations are easily generalized to a uniaxial material. In addition to the field fluctuations described by Eq. (2.7), there are field fluctuations representing thermal deviations from the solution of London equation, which are neglected here.

In the chosen gauge $(\boldsymbol{\nabla} \cdot \mathbf{A}=0)$ the longitudinal part of the supercurrent is simply determined by the phase in the usual way, 


$$
\boldsymbol{\nabla} \cdot \mathbf{j}_{s}=\frac{c \phi_{0}}{4 \pi \lambda^{2}} \nabla^{2} \theta .
$$

Vortices provide a source of vorticity for the supercurrent, as the curl of Eq. (2.7) gives

$$
\boldsymbol{\nabla} \times \mathbf{j}_{s}=-\frac{c}{4 \pi \lambda^{2}}\left(\mathbf{b}-\phi_{0} \mathbf{T}\right),
$$

where $\mathbf{T}$ is the vortex density vector,

$$
\mathbf{T}(\mathbf{r}, t)=\frac{1}{2 \pi} \nabla \times \nabla \theta .
$$

We note that the curl of $\nabla \theta$ is nonzero in the presence of vortices. By combining Eq. (2.9) with Eq. (2.2), we immediately obtain the London equation determining the field due to the vortices,

$$
\mathbf{b}-\lambda^{2} \nabla^{2} \mathbf{b}=\phi_{0} \mathbf{T},
$$

To obtain the contribution to the electric field from the vortex degrees of freedom, we differentiate Eq. (2.7) with respect to time and use $\mathbf{e}=-\frac{1}{c} \partial_{t} \mathbf{A}-\boldsymbol{\nabla} \phi$, with the result

$$
\mathbf{e}=-\boldsymbol{\nabla} \phi+\frac{4 \pi \lambda^{2}}{c^{2}} \partial_{t} \mathbf{j}_{s}-\frac{\phi_{0}}{c} \partial_{t} \boldsymbol{\nabla} \theta .
$$

The vortex part of the electric field is then given by

$$
\mathbf{e}^{v}-\lambda^{2} \nabla^{2} \mathbf{e}^{v}=\frac{\phi_{0}}{2 c} \epsilon_{\alpha \beta \gamma} Q_{\beta \gamma},
$$

where

$$
\frac{1}{2} \epsilon_{\alpha \beta \gamma} Q_{\beta \gamma}(\mathbf{r}, t)=-\left(\partial_{t} \partial_{\alpha} \theta(\mathbf{r}, t)\right)^{T} .
$$

The antisymmetric tensor $Q_{\beta \gamma}$ as defined as in Eq. (2.14) describes the vortex current. It is precisely the vortex flux tensor as with the definition (2.14) the vortex density vector $\mathbf{T}$ satisfies the exact conservation law (conservation of vorticity)

$$
\partial_{t} T_{\alpha}+\partial_{\beta} Q_{\alpha \beta}=0 .
$$

The focus of the present paper is on the flux-line liquid regime of vortex matter. In order to take advantage of the vast phenomenology developed for describing long-wavelength static and dynamical properties of dense liquids with partcle-like or polymer-like degrees of freedom, we will focus our attention below on the vortex degrees of freedom, rather that on the fields $\mathbf{b}$ and $\mathbf{e}$. When the $z$ direction is chosen along the external field $\mathbf{H}$, vortex configurations are conveniently parametrized in terms of a set of $N$ single-valued functions $\mathbf{r}_{n}(z)$, which specify the position of the $n$-th vortex line in the $x y$ plane as it traverses the sample along the field direction. The three-dimensional position of each flux line is then $\mathbf{R}_{n}(z)=\left[\mathbf{r}_{n}(z), z\right]$ and the vortex density vector and flux tensor are given by,

$$
\begin{aligned}
& \mathbf{T}(\mathbf{r}, t)=\sum_{n=1}^{N} \frac{\partial \mathbf{R}_{n}}{\partial z} \delta\left(\mathbf{r}-\mathbf{r}_{n}(z, t)\right), \\
& Q_{\alpha \beta}(\mathbf{r}, t)=\epsilon_{\alpha \beta \gamma} \sum_{n=1}^{N}\left(\partial_{z} \mathbf{R}_{n} \times \mathbf{v}_{n}\right)_{\gamma} \delta\left(\mathbf{r}-\mathbf{r}_{n}(z, t)\right),
\end{aligned}
$$

with $\mathbf{r}=\left(\mathbf{r}_{\perp}, z\right)$ and $\mathbf{v}_{n}(z, t)=\partial_{t} \mathbf{r}_{n}$ the vortex velocity. The three-dimensional vortex density vector is also often written as

$$
\mathbf{T}=(\mathbf{t}, n),
$$

where $n$ is the local areal density of vortices and $\mathbf{t}$ describes the local tilt of vortex lines away from the $z$ direction. Neglecting spatial inhomogeneities on scales $\leq \lambda$, Eq. (2.11) gives 


$$
\begin{aligned}
& b_{z}(\mathbf{r}, t)=\phi_{0} n(\mathbf{r}, t), \\
& \mathbf{b}_{\perp}(\mathbf{r}, t)=\phi_{0} \mathbf{t}(\mathbf{r}, t) .
\end{aligned}
$$

Similarly, the vortex flux tensor $Q_{\alpha \beta}$ can be written as

$$
\left(\begin{array}{lll}
Q_{x x} & Q_{x y} & Q_{x z} \\
Q_{y x} & Q_{y y} & Q_{y z} \\
Q_{z x} & Q_{z y} & Q_{z z}
\end{array}\right)=\left(\begin{array}{ccc}
0 & n_{0} V & -n_{0} v_{x} \\
-n_{0} V & 0 & -n_{0} v_{y} \\
n_{0} v_{x} & n_{0} v_{y} & 0
\end{array}\right)
$$

where $\mathbf{v}$ represent the flow velocity of vortices moving in the $x y$ plane and $n_{0} V=\frac{1}{2} \epsilon_{i j} Q_{i j}$ is the number of vortex segments aligned with the $x$ direction crossing an area normal to the $y$ direction per unit area and per unit time, with

$$
\begin{aligned}
& n_{0} \mathbf{v}(\mathbf{r}, t)=\sum_{n=1}^{N} \mathbf{v}_{n} \delta\left(\mathbf{r}-\mathbf{r}_{n}(z, t)\right) \\
& n_{0} V(\mathbf{r}, t)=\sum_{n=1}^{N} \hat{\mathbf{z}} \cdot\left(\partial_{z} \mathbf{r}_{n} \times \mathbf{v}_{n}\right) \delta\left(\mathbf{r}-\mathbf{r}_{n}(z, t)\right) .
\end{aligned}
$$

We have explicitly linearized in the fluctuations as we are only interested in linearized hydrodynamic equations. Neglecting again nonlocalities on length scales $\sim \lambda$, Eq. (2.13) yields

$$
e_{\alpha}^{v}=\frac{\phi_{0}}{2 c} \epsilon_{\alpha \beta \gamma} Q_{\beta \gamma}
$$

or

$$
\begin{aligned}
& e_{z}^{v}=\frac{\phi_{0}}{2 c} \epsilon_{i j} Q_{i j}=\frac{n_{0} \phi_{0}}{c} V, \\
& e_{i}^{v}=-\frac{\phi_{0}}{c} \epsilon_{i j} Q_{z j}=-\frac{n_{0} \phi_{0}}{c} \epsilon_{i j} v_{j} .
\end{aligned}
$$

We are interested here in the long-wavelength properties of flux-line liquids on scales large compared to $\lambda$ and to the average intervortex spacing, $a_{0}=\sqrt{\phi_{0} / B_{0}}$, with $B_{0}$ the equilibrium mean value of the field, $\langle\mathbf{b}\rangle=\hat{\mathbf{z}} B_{0}$. For this purpose we can abandon the description in terms of the microscopic vortex degrees of freedom $\mathbf{R}_{n}(z)$ in terms of coarse-grained or hydrodynamic fields describing fluctuations in the conserved variables of the system and corresponding to those collective degrees of freedom whose relaxation rate vanishes in the long wavelength limit. For the vortex liquid the relevant conserved variables are the three components of the vortex density, $\mathbf{T}(\mathbf{r}, t)=(\mathbf{t}, n)$. The coarse-grained vortex density is defined as in Eq. (2.16), with the $\delta$-function replaced by a smeared-out twodimensional $\delta$-function, $\delta_{B Z}\left(\mathbf{r}_{\perp}\right)$, with a finite spatial extent of the order of the inverse of the Brillouin zone boundary, $k_{B Z}=\sqrt{4 \pi n_{0}}$,

$$
\delta_{B Z}\left(\mathbf{r}_{\perp}\right)=\frac{1}{A} \sum_{q \perp \leq k_{B Z}} e^{-i \mathbf{q}_{\perp} \cdot \mathbf{r}_{\perp}} .
$$

As discussed elsewhere, the long wavelength equilibrium properties of vortex liquids can be described in terms of a coarse-grained free energy that fully incorporates the nonlocality of the intervortex interaction. Such a free energy can be derived by explicit coarse-graining of the microscopic vortex energy 31 or can be written down phenomenologically using familiar ideas from liquid state physics. It takes the form of an expansion in the fluctuations of the hydrodynamic fields from their equilibrium values and of the corresponding gradients. Retaining only terms quadratic in the fluctuations, it is given by

$$
F_{L}=\frac{1}{2 n_{0}^{2}} \int_{\mathbf{r}} \int_{\mathbf{r}^{\prime}}\left[c_{L}\left(\mathbf{r}-\mathbf{r}^{\prime}\right) \delta n(\mathbf{r}) \delta n\left(\mathbf{r}^{\prime}\right)+c_{44}\left(\mathbf{r}-\mathbf{r}^{\prime}\right) \mathbf{t}(\mathbf{r}) \cdot \mathbf{t}\left(\mathbf{r}^{\prime}\right)\right]
$$

where $\delta n-\bar{B} n-n_{0}$ and $c_{L}$ and $c_{44}$ are the nonlocal compressional and tilt elastic moduli of the flux liquid, given elsewhere. 13

The vortex density vector, $\mathbf{T}=(\mathbf{t}, n)$, satisfies an exact conservation law, Eq. 2.15), or, in terms of $n$ and $\mathbf{t}$,

$$
\begin{aligned}
& \partial_{t} n+\nabla \cdot n_{0} \mathbf{v}=0, \\
& \partial_{t} t_{i}+\partial_{j} Q_{i j}=\partial_{z} n_{0} v_{i},
\end{aligned}
$$


with the condition $\boldsymbol{\nabla} \cdot \mathbf{T}=0$, or

$$
\partial_{z} n+\nabla_{\perp} \cdot \mathbf{t}=0
$$

ensuring that flux lines do not start nor stop inside the sample.

To obtain a closed set of hydrodynamic equations it is necessary to specify the constitutive equations that express the flux tensor $Q_{\alpha \beta}$ in terms of the density field and its gradients. Since the vortex dynamics is overdamped, the constitutive equation is simply obtained requiring that the net force on each local volume of flux liquid vanishes. Its form is analogous to that of Eq. (2.6) and it is given by

$$
\tilde{\gamma}_{\mu \nu, \lambda \rho} Q_{\lambda \rho}=\tilde{\eta}_{\mu \nu, \alpha \beta, \lambda \rho} \partial_{\alpha} \partial_{\beta} Q_{\lambda \rho}+\partial_{\mu} \frac{\delta F_{L}}{\delta T_{\nu}}-\partial_{\nu} \frac{\delta F_{L}}{\delta T_{\mu}}+\frac{\phi_{0}}{c} \epsilon_{\mu \nu \lambda} J_{\lambda} .
$$

The first term on the left hand side of Eq. (2.30) represents the frictional force per unit length on the vortex liquid, with $\tilde{\gamma}_{\mu \nu, \lambda \rho}$ a friction tensor. The second term describes viscous forces from intervortex interaction and entanglement, with $\tilde{\eta}_{\mu \nu, \alpha \beta, \lambda \rho}$ the viscosity tensor. The third term contains Magnus and pressure forces arising from vortex density gradients and the last one is simply the Lorentz force due to an external current, $\mathbf{J}$.

Neglecting for simplicity transverse drag forces associated with the Hall effect, there are two independent components of the friction tensor and six viscosity coefficients. It is useful to rewrite the constitutive equations in terms of the in-plane vortex flow velocity $\mathbf{v}$ and the tilt current $V$,

$$
\begin{gathered}
\gamma_{\perp} \mathbf{v}=\left[\eta \nabla_{\perp}^{2}+\eta_{z} \partial_{z}^{2}\right] \mathbf{v}+\eta_{b} \nabla_{\perp}\left(\nabla_{\perp} \cdot \mathbf{v}\right)-\eta_{x}\left(\hat{\mathbf{z}} \partial_{z} \times \nabla_{\perp}\right) V \\
-n_{0}\left(\nabla_{\perp} \frac{\delta F_{L}}{\delta n}-\partial_{z} \frac{\delta F_{L}}{\delta \mathbf{t}}\right)-\frac{n_{0} \phi_{0}}{c} \hat{\mathbf{z}} \times \mathbf{J} . \\
\gamma_{\|} V=\left[\eta^{t} \nabla_{\perp}^{2}+\eta_{z}^{t} \partial_{z}^{2}\right] V-\eta_{x} \hat{\mathbf{z}} \partial_{z} \cdot\left(\nabla_{\perp} \times \mathbf{v}\right)+n_{0} \hat{\mathbf{z}} \cdot\left(\nabla_{\perp} \times \frac{\delta F_{L}}{\delta \mathbf{t}}\right)+\frac{n_{0} \phi_{0}}{c} J_{z} .
\end{gathered}
$$

The constitutive equations (2.31) and (2.32) are now written in terms of forces per unit volume. The coefficients $\gamma_{\perp}$ and $\gamma_{\|}$have dimension of friction per unit volume, as usual, and $\eta, \eta_{z}, \eta_{b}, \eta^{t}, \eta_{z}^{t}$ and $\eta_{x}$ have dimensions of fluid viscosities.

The six viscosity coefficients appearing in Eqs. 2.31) and (2.32) are related to the six components of the nonlocal conductivity discussed by Mou et al.28 The precise relationship is displayed in Appendix A. Equations (2.27-2.29) and $(2.31,2.32$ provide a closed set of hydrodynamic equations to describe the response of vortex liquids to external currents and fields. These equations generalize equations written down earlier by us 24 and by others 32,28 . by incorporating all pressure forces, including those from vortex segments that are not parallel to the external field direction, all nonlocal viscous forces, as well as external forces due to driving currents aligned with the external field. In the next section we will show how predictions for the singular behavior of for the six viscosities near a continuous phase transition from the vortex liquid to a glassy state can be obtained by simple scaling arguments.

\section{SCALING THEORY}

The divergence of pinning energy barriers for vanishing driving currents underlies both the theories of the Bose glass and the vortex glass transitions, which are expected to be continuous. The properties near the transition have therefore been described by a scaling theory in terms of divergent lengths and time scales. A scaling theory for the transition from a vortex liquid to an isotropic vortex glass was developed by Fisher, Fisher and Huse $\mathrm{G}$ and then adapted by Nelson and Vinokur to the transition to the anisotropic Bose glass 14 Here we summarize this scaling theory in a unified way that applies to both transitions by keeping track of the anisotropy exponent $\zeta$ that has value $\zeta=2$ for the Bose glass and $\zeta=1$ for the vortex glass. Of course the values of the other critical exponents are also different in the two cases.

The (Gibbs) free energy density $g$ of the vortex array is a function of temperature $T$ and external field, $\mathbf{H}=$ $\hat{\mathbf{z}} H_{\|}+\mathbf{H}_{\perp}$, with $g=g\left(T, H_{\perp}, H_{\|}\right)$. Here $H_{\|}=H_{0}+\delta H_{\|}$, where $H_{0}$ is the field responsible for setting up the vortex state and the flux lines are on average aligned with the $z$ direction. As predicted by the renormalization group, the singular part of the free energy density, $g_{s}$, is assumed to obey a homogeneity relation at the transition where temperature and the external fields are rescaled by different factors,

$$
g_{s}\left(t, H_{\perp}, \delta H_{\|}\right)=l^{-(d-1+\zeta)} g_{s}\left(l^{1 / \nu} t, l^{\lambda_{\perp}} H_{\perp}, l^{\lambda_{\|}} \delta H_{\|}\right)
$$


for arbitrary length rescaling parameter, $l$. Here $t$ is the reduced temperature $t=\left|T-T_{G}\right| / T_{G}$, with $T_{G}$ either the Bose glass or the vortex glass transition temperature, and $d$ denotes the system dimensionality. The case of interest here is $d=3$, corresponding to a bulk superconductor. In this section $\lambda_{\perp}$ and $\lambda_{\|}$denote critical exponents, not to be confused with penetration lengths. Making the usual choice $l=t^{-\nu} \sim \xi_{\perp}$, dimensional analysis will allows us to relate physical quantities to the diverging correlation lengths $\xi_{\perp}$ and $\xi_{\|} \sim \xi_{\perp}^{\zeta}$, as described in Ref. 6, 14, 17, 18, 20,21.

The local field in the superconductor is given by

$$
\mathbf{b}=-4 \pi \frac{\delta g}{\delta \mathbf{H}}
$$

and therefore scales according to

$$
\begin{aligned}
b_{\perp} & \sim l^{-\left(d-1+\zeta+\lambda_{\perp}\right)}, \\
b_{\|} & \sim l^{-\left(d-1+\zeta+\lambda_{\|}\right)} .
\end{aligned}
$$

The longitudinal and transverse components of the response function are

$$
\begin{aligned}
& \delta_{i j}\left(1+4 \pi \chi_{\perp}\right)=\frac{\delta^{2} g_{s}}{\delta H_{\perp i} \delta H_{\perp j}}=\delta_{i j} \frac{n_{0}^{2} \phi_{0}^{2}}{c_{44}}, \\
& 1+4 \pi \chi_{\|}=\frac{\delta^{2} g_{s}}{\delta H_{\|}^{2}}=\frac{n_{0}^{2} \phi_{0}^{2}}{c_{11}},
\end{aligned}
$$

where $\chi_{\perp}$ and $\chi_{\|}$are the components of the magnetic susceptibility and $c_{44}$ and $c_{11}$ the tilt and compressional elastic moduli of the vortex array. The scaling of the susceptibilities is then given by

$$
\begin{aligned}
& \chi_{\perp} \sim \frac{1}{c_{44}} \sim l^{-\left(d-1+\zeta+2 \lambda_{\perp}\right)}, \\
& \chi_{\|} \sim \frac{1}{c_{11}} \sim l^{-\left(d-1+\zeta+2 \lambda_{\|}\right)},
\end{aligned}
$$

At the vortex glass transition the response of the vortex array is rotationally invariant and both susceptibilities are expected to remain finite. This requires

$$
\lambda_{\perp}^{V G}=\lambda_{\|}^{V G}=-(d-1+\zeta) / 2 .
$$

The scaling of the local fields at the vortex glass transition is then

$$
b_{\perp} \sim b_{\|} \sim \frac{1}{\xi_{\perp}^{(d-1) / 2} \xi_{\|}^{1 / 2}} \sim t^{3 \nu / 2},
$$

where the last equality applies for $d=3$.

At the Bose glass transition, in contrast, the tilt modulus is expected to diverge as $c_{44} \sim l^{\zeta}$, while $c_{11}$ remains finite. In this case the response of the system is anisotropic, with

$$
\begin{aligned}
& \lambda_{\perp}^{B G}=-(d-1) / 2, \\
& \lambda_{\|}^{B G}=-(d-1+\zeta) / 2,
\end{aligned}
$$

and the fields scale as

$$
\begin{aligned}
& b_{\perp} \sim \frac{1}{\xi_{\perp}^{(d-1) / 2} \xi_{\|}} \sim t^{3 \nu}, \\
& b_{\|} \sim \frac{1}{\xi_{\perp}^{(d-1) / 2} \xi_{\|}^{1 / 2}} \sim t^{2 \nu},
\end{aligned}
$$

where again the last equality in each of Eqs. (3.13) applies for $d=3$.

To determine the scaling of fields and currents that control the resistive properties of the vortex array, we note that gauge invariance of the Ginzburg-Landau theory requires that the fluctuating vector potential scales according to 


$$
\begin{aligned}
& A_{\perp} \sim \xi_{\perp}^{-1}, \\
& A_{z} \sim \xi_{\|}^{-1} .
\end{aligned}
$$

The scaling of currents and fields is immediately obtained from their definitions, $\mathbf{J}=\partial f / \partial \mathbf{A}$ and $\mathbf{E}=-(1 / c) \partial \mathbf{A} / \partial t$, where we denote by $\mathrm{E}$ the macroscopic (spatially-averaged) field from vortex motion. It is given by,

$$
\begin{aligned}
& J_{\perp} \sim \xi_{\perp}^{2-d} \xi_{\|}^{-1}, \\
& J_{\|} \sim \xi_{\perp}^{1-d},
\end{aligned}
$$

and

$$
\begin{aligned}
& E_{\perp} \sim \xi_{\perp}^{-(1+z)}, \\
& E_{\|} \sim \xi_{\|}^{-1} \xi_{\perp}^{-z} .
\end{aligned}
$$

A scaling ansatz for the IV characteristic can be written down based on the structure of the RG flows as

$$
\begin{aligned}
& E_{\perp}\left(T, J_{\perp}\right)=l^{-(1+z)} E_{\perp}\left(l^{1 / \nu} t, \frac{l^{(d-2+\zeta)} J_{\perp}}{c k_{B} T}\right), \\
& E_{\|}\left(T, J_{\|}\right)=l^{-(\zeta+z)} E_{\|}\left(l^{1 / \nu} t, \frac{l^{d-1} J_{\|}}{c k_{B} T}\right) .
\end{aligned}
$$

With the choice $l=t^{-\nu} \sim \xi_{\perp}(T)$, we obtain

$$
\begin{aligned}
& E_{\perp}\left(1, J_{\perp}\right)=\xi_{\perp}^{-(1+z)} \mathcal{E}_{\perp}^{ \pm}\left(\frac{\xi_{\perp}^{d-2} \xi_{\|} J_{\perp}}{c k_{B} T}\right), \\
& E_{\|}\left(1, J_{\|}\right)=\xi_{\|}^{-1} \xi_{\perp}^{-z} \mathcal{E}_{\|}^{ \pm}\left(\frac{\xi_{\perp}^{d-1} J_{\|}}{c k_{B} T}\right)
\end{aligned}
$$

where $\mathcal{E}_{\perp, \|}^{ \pm}$are scaling functions. From these one can obtain various results described in the literature. In particular, by linearizing Eqs. (3.17) and (3.18) in the ohmic regime, one finds that both components of the linear flux liquid resistivity vanish as the transition is approached from the liquid side, according to,

$$
\begin{aligned}
& \rho_{\perp}(T)=\left(\frac{n_{0} \phi_{0}}{c}\right)^{2} \frac{1}{\gamma_{\perp}(T)} \sim \xi_{\|} \xi_{\perp}^{-(z+3-d)} \sim\left|T-T_{B G}\right|^{\nu(3+z-d-\zeta)}, \\
& \rho_{\|}(T)=\left(\frac{n_{0} \phi_{0}}{c}\right)^{2} \frac{1}{\gamma_{\|}(T)} \sim \xi_{\|}^{-1} \xi_{\perp}^{-(z+1-d)} \sim\left|T-T_{B G}\right|^{\nu(1+z-d+\zeta)} .
\end{aligned}
$$

Equivalently, Eqs. (3.21) and (3.22) give the scaling exponents controlling the divergence of the longitudinal and transverse friction coefficients, $\gamma_{\|}(T)$ and $\gamma_{\perp}(T)$.

Dimensional analysis of the hydrodynamic equations now allows us to extract predictions for the scaling of the six viscosity coefficients at the transition. The scaling dimension of the fluctuating density and tilt fields $n$ and $\mathbf{t}$ is the same as that of the components of the local field $\mathbf{b}$ and therefore is obtained immediately from Eqs. (3.10) and (3.13) for the vortex and Bose glass, respectively. We scale all length and time scales in the hydrodynamic equations with the correlation lengths $\xi_{\perp}$ and $\xi_{\|}$, using the scaling dimension just discussed for the various physical quantities appearing in these equations. By requiring the equations to be scale invariant near the transition, we can then read off the critical scaling of the viscosity coefficients,

$$
\begin{aligned}
\eta & \sim \xi_{\perp}^{2} \gamma_{\perp} \sim \xi_{\perp}^{z+5-d} / \xi_{\|}, \\
\eta_{b} & \sim \xi_{\perp}^{2} \gamma_{\perp} \sim \xi_{\perp}^{z+5-d} / \xi_{\|}, \\
\eta_{z} & \sim \xi_{\|}^{2} \gamma_{\perp} \sim \xi_{\perp}^{z+3-d} \xi_{\|}, \\
\eta^{t} & \sim \xi_{\perp}^{2} \gamma_{\|} \sim \xi_{\perp}^{z+3-d} \xi_{\|}, \\
\eta_{z}^{t} & \sim \xi_{\|}^{2} \gamma_{\|} \sim \xi_{\perp}^{z+1-d} \xi_{\|}^{3}, \\
\eta_{x} & \sim \xi_{\perp}^{2} \gamma_{\|} \sim \xi_{\|}^{2} \gamma_{\perp} \sim \xi_{\perp}^{z+3-d} \xi_{\|} .
\end{aligned}
$$

The critical exponents for all the friction and viscosity coefficients are summarized in Table 1. 


\section{SCALING AND HYDRODYNAMICS IN CONFINED GEOMETRIES}

The flow of vortex liquids in confined geometries provides a powerful experimental tool to probe the shear resistance of vortex matter. Experiments of this type have been carried out by the group of Peter Kes in thin superconducting films.22. 25 In this two-dimensional case the vortex array is expected to melt from a solid into a liquid via the dislocation unbinding mechanism. 29 The shear modulus of the lattice vanishes continuously at the transition and is replaced by a shear viscosity in the liquid phase. The shear viscosity diverges as the transition is approached from the liquid side. In order to probe this behavior, Theunissen et al. studied the flow of a two-dimensional vortex solid through narrow channels in a double-layer device, consisting of a weakly pinning amorphous $\mathrm{Nb}_{3} \mathrm{Ge}$ bottom layer, covered with a strongly pinning $N b N$ thick layer. Using nanolithography, parallel channels were etched through the $N b N$ layer into the $N b G e$ layer. By applying a current normal to the direction of the channels, vortices where then forced to flow along the weakly pinning channels, while the $N b N$ regions provide the strong-pinning boundaries.22 By fitting their data to the form for the flux-flow resistivity of a vortex liquid in a channel obtained earlier by us via a simple hydrodynamic model24, these authors were able to extract the temperature dependence of the shear viscosity near melting, finding a behavior in agreement with the predictions of the theory of two-dimensional melting.20

As discussed in the Introduction, patterned irradiation of cuprate superconductors with heavy ions allows for experiments similar in spirit to the one carried out by Theunissen et al.22. These experiments can probe the growing shear viscosity of the vortex array near a continuous glass transition and may shed some light on the connection between static and dynamical properties of these glassy systems.

Scaling theory can again be used to determine the temperature dependence of fields and currents in this spatially inhomogeneous situation arising from the confined geometry. A generalized homogeneity relation for the local electric field $e_{\perp}$ perpendicular to any correlated disorder from flux motion at position $x$ in a channel of thickness $L$ takes the form

$$
e_{\perp}\left(T, J_{\perp}, x, L\right)=l^{-(1+z)} e_{\perp}\left(l^{1 / \nu} t, \frac{l^{\nu(1+\zeta)} J_{\perp} \phi_{0}}{c k_{B} T}, \frac{x}{l}, \frac{L}{l}\right)
$$

By choosing again $l=t^{-\nu_{\perp}} \sim \xi_{\perp}(T)$ we obtain

$$
e_{\perp}\left(T, J_{\perp}, x, L\right)=\xi_{\perp}^{-(1+z)} \mathcal{E}_{\perp}\left(\frac{\xi_{\perp} \xi_{\|} J_{\perp} \phi_{0}}{c k_{B} T}, \frac{x}{\xi_{\perp}}, \frac{L}{\xi_{\perp}}\right) .
$$

In the entangled flux liquid the response is linear at small current. Upon expanding the right hand side of Eq. (4.2) we obtain for $J_{\perp} \rightarrow 0$

$$
e_{\perp}\left(J_{\perp} \rightarrow 0, x, L\right) \simeq \rho_{\perp}^{0}\left(\frac{\xi_{\perp}}{a_{0}}\right)^{2-z} J_{\perp} \mathcal{F}\left(x / \xi_{\perp}, L / \xi_{\perp}\right)
$$

where $\rho_{\perp}^{0}=\left(n_{0} \phi_{0} / c\right)^{2}\left(1 / \gamma_{\perp}^{0}\right)$ is the Bardeen-Stephen resistivity of noninteracting flux lines, with $\gamma_{\perp}^{0}$ a bare friction. A scaling form for the resistivity $\rho_{\perp}(T, L)=\Delta V /\left(L J_{\perp}\right)$, with $\Delta V$ the net voltage drop across the channel, is easily obtained by integrating Eq. (4.3), with the result.

$$
\rho_{\perp}(T, L)=\rho_{\perp}(T) f\left(L / \xi_{\perp}\right)
$$

with $f(x)=\frac{1}{x} \int_{x / 2}^{x / 2} d u \mathcal{F}(u, x)$ a scaling function and $\rho_{\perp}(T)$ the bulk flux liquid resistivity given in Eq. (1.5). Here $\gamma_{\perp}$ is the renormalized friction coefficient that incorporates the growing Bose glass correlations near $T_{B G}, \gamma_{\perp}=\gamma_{\perp}^{0}\left(\frac{\xi_{\perp}}{a_{0}}\right)^{z-2}$, and diverges at the transition as $\gamma_{\perp} \sim\left|T-T_{B G}\right|^{-\nu(z-2)}\left\lfloor\right.$. 4 . For $L \gg \xi_{\perp}$, the channel geometry has no effect and one must recover the bulk result. This requires $f(x \gg 1) \sim 1$.

The scaling function $\mathcal{F}$ can be determined by assuming that the long wavelength electric field of Eq. (4.3) is described by the set of hydrodynamic equations discussed in Ref. 24. For simple geometries where the current is applied in the $a b$ plane and the flow is spatially homogeneous in the $z$ direction, these reduce to the single equation for the coarse-grained flux liquid flow velocity $\mathbf{v}(\mathbf{r})$ given in Eq. (1.6). Intervortex interaction at the Bose-glass boundaries yields "adhesion" of the flux liquid to these boundaries, forcing the flux flow velocity to vanish or become very small. In the hydrodynamic model this translates into a no-slip boundary condition for the flux liquid velocity at the boundaries. By preventing the free flow of flux liquid, the Bose glass boundaries can significantly decrease the macroscopic flux-flow resistivity of the superconductor. The electric field profile is obtained by solving Eq. (1.7) for the appropriate geometry and with suitable boundary conditions. 


\section{A. Channel geometry}

The solution of the hydrodynamic equation for the simple channel gepmetry sketched in Fig. 2, with a homogeneous current $\mathbf{J}=-\hat{\mathbf{x}} J_{\perp}$ applied across the channel has been given elsewhere 2423 and is displayed here for completeness. It is given by

$$
e_{\perp}(x, L)=\rho_{\perp} J_{\perp}\left[1-\frac{\cosh \left(x / \xi_{\perp}\right)}{\cosh \left(L / 2 \xi_{\perp}\right)}\right] .
$$

Upon comparing Eq. (4.5) to Eq. (4.3), we identify the quantity in square brackets in Eq. (4.5) with the scaling function $\mathcal{F}$. The scaling form for the resistivity is obtained by integrating Eq. (4.5), with the result

$$
\rho_{\perp L}(T, L)=\rho_{\perp}(T)\left[1-\frac{2 \xi_{\perp}}{L} \tanh \left(\frac{L}{2 \xi_{\perp}}\right)\right] .
$$

If $\xi_{\perp} \ll L$, we recover the bulk result given by Eq. (3.21),

$$
\rho_{\perp L}(T, L) \simeq \rho_{\perp}(T) \sim t^{\nu(z-\zeta)} .
$$

Near the transition, however, $\xi_{\perp} \gg L$, and the resistivity depends on the channel width and is controlled by the shear viscosity, with

$$
\rho_{\perp L}(T, L) \simeq \frac{\rho_{\perp} L^{2}}{12 \xi_{\perp}^{2}}=\left(\frac{n_{0} \phi_{0}}{c}\right)^{2} \frac{L^{2}}{12 \eta(T)} \sim L^{2} t^{\nu(z+2-\zeta)} .
$$

The strong divergence of the viscosity implicit in Eq. (4.8) is precisely the kind of behavior expected at a liquid-glass transition. The Bose glass transition is an example of a glass transition that is well understood theoretically and where precise predictions are available.

\section{B. Corbino disk}

Experiments in patterned geometries near the Bose glass transformation provide an exciting opportunity to probe viscous behavior near a second order glass transition. Other patterned geometries can be designed and used to probe some or all of the other viscosity coefficients. Of particular interest is the Corbino disk geometry, recently used by López et al. for defect-free materials 30 to reduce boundary effects in the flux flow measurements. This geometry was briefly discussed in 23 and is sketched in Fig. 4.

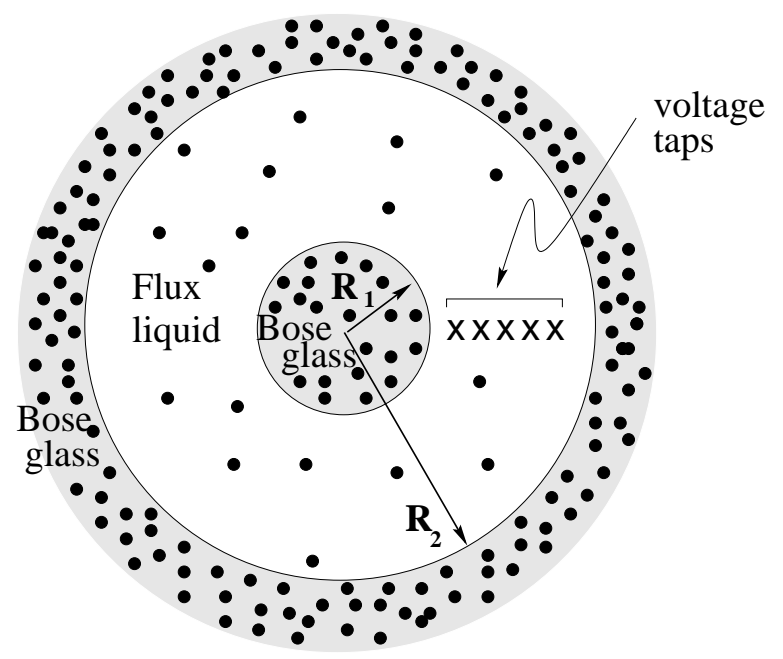

Figure 4. Top view of the Corbino disk geometry with Bose glass contacts. The magnetic field is out of the page. The vortex array is in the Bose glass state in the inner and outer densely dotted regions and in the flux liquid state in the weakly irradiated annular region. A radial driving current drives flux motion in the azimuthal direction. 
In 23 we proposed the fabrication of a Corbino disk with Bose glass inner and outer contacts. The annular region corresponding to $R_{1} \leq r \leq R_{2}$ is in the vortex liquid state. A constant current $I$ is injected at the inner boundary of the cylindrical flux liquid region and extracted at the outer boundary. The resulting current density is radial, with

$$
\mathbf{J}=\frac{I}{2 \pi W} \frac{\hat{\mathbf{r}}}{r}
$$

with $W=R_{2}-R_{1}$ the width of the annular flux liquid channel. This current induces tangential flow of the vortices, which in turn yields a radial voltage drop that can be probed by a suitable set of voltage taps, placed as sketched in Fig. 4. If the intervortex spacing $a_{0}$ in the flux liquid is small compared to the size $R_{2}-R_{1}$ of the annular channel where flux flow occurs, the flow can be described by the hydrodynamic equation (1.7). Using cylindrical coordinates with the $z$ axis directed along the direction of the external field $\mathbf{H} \| c$, the vortex flow velocity is in the azimuthal direction, $\mathbf{v}=v_{\phi}(r) \hat{\boldsymbol{\phi}}$, corresponding to a radial field, $e_{r}(r)=\frac{n_{0} \phi_{0}}{c} v_{\phi}(r)$. The nonlocal Ohm's law takes the form

$$
\xi_{\perp}^{2} \frac{1}{r} \frac{d}{d r} r \frac{d}{d r} e_{r}(r)-\left(1+\frac{\xi_{\perp}^{2}}{r^{2}}\right) e_{r}(r)=-\frac{\alpha_{L}}{\gamma_{\perp} r}
$$

where $\alpha_{L}=n_{0} \phi_{0} I /(2 \pi W c)$ controls the strength of the Lorentz force. The general solution of Eq. 4.10 is given by

$$
e_{r}(r)=\frac{\rho_{\perp} I}{2 \pi W \xi_{\perp}}\left[\frac{\xi_{\perp}}{r}+c_{1} I_{1}\left(r / \xi_{\perp}\right)+c_{2} K_{1}\left(r / \xi_{\perp}\right)\right]
$$

where $I_{1}$ and $K_{1}$ are Bessel functions. The constants $c_{1}$ and $c_{2}$ are determined by requiring the liquid flow velocity, and therefore the electric field, to vanish at the boundaries with the Bose glass regions, $e_{r}\left(R_{1}\right)=e_{r}\left(R_{2}\right)=0$, with the result,

$$
\begin{aligned}
& c_{1}=\frac{K_{1}\left(\rho_{2}\right) / \rho_{1}-K_{1}\left(\rho_{1}\right) / \rho_{2}}{K_{1}\left(\rho_{1}\right) I_{1}\left(\rho_{2}\right)-K_{1}\left(\rho_{2}\right) I_{1}\left(\rho_{1}\right)} \\
& c_{2}=\frac{I_{1}\left(\rho_{1}\right) / \rho_{2}-I_{1}\left(\rho_{2}\right) / \rho_{1}}{K_{1}\left(\rho_{1}\right) I_{1}\left(\rho_{2}\right)-K_{1}\left(\rho_{2}\right) I_{1}\left(\rho_{1}\right)},
\end{aligned}
$$

where $\rho_{1,2}=R_{1,2} / \xi_{\perp}$. As in the channel problem, spatial inhomogeneities are controlled by the in-plane correlation length $\xi_{\perp}$. The field profile for a few values of $\xi_{\perp}$ in a micron-scale sample is shown in Fig. 5 .

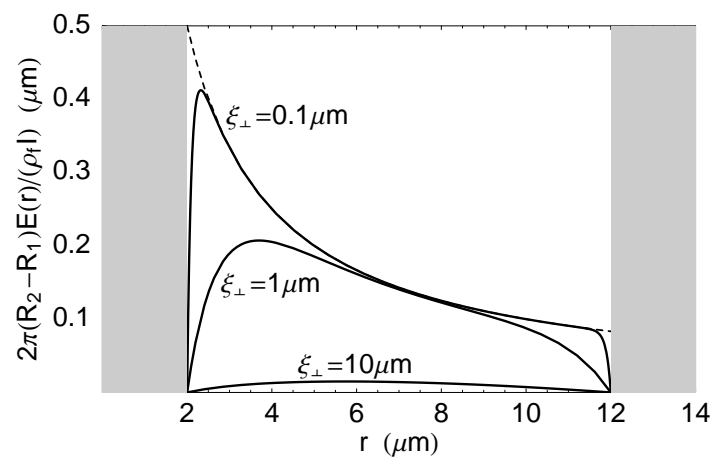

Figure 5. The electric field profile for the Corbino disk geometry, with $R_{1}=2 \mu m$ and $R_{2}=12 \mu m$, with an annular width $R_{2}-R_{1}=10 \mu \mathrm{m}$. The vertical axis represents the reduced field $2 \pi e_{r}(r) /\left(\rho_{\perp} I\right)$ in $\mu \mathrm{m}$. The dashed line is the electric field for vanishing viscosity, $e_{r}^{0}(r)=\frac{\rho_{\perp} I}{2 \pi\left(R_{2}-R_{1}\right)} \frac{1}{r}$. The shaded regions represent the Bose glass contacts.

The field nonlocality arising from the growing correlation length near the Bose glass transition can be probed experimentally by measuring the voltage profile across the annular channel. A series of equally spaced contacts are placed radially, as shown in Fig. 5, at positions $r_{n}=R_{1}+n d$, for $n=1,2, \ldots, N$. For the magnetic fields and temperatures of interest, the spacing $d$ of the contacts is large compared to the intervortex spacing $a_{0}$, so that hydrodynamics can be applied. The voltage drop across two successive contacts is obtained immediately by integrating Eq. (4.11) and it is given by 


$$
\begin{aligned}
& \Delta V\left(r_{n+1}, r_{n}\right)=\int_{r_{n}}^{r_{n+1}} d r e_{r}(r) \\
& =\frac{\rho_{\perp} I}{2 \pi W}\left\{\ln \left(r_{n+1} / r_{n}\right)+c_{1}\left[I_{0}\left(r_{n+1} / \xi_{\perp}\right)-I_{0}\left(r_{n} / \xi_{\perp}\right)\right]\right. \\
& \left.\quad-c_{2}\left[K_{0}\left(r_{n+1} / \xi_{\perp}\right)-K_{0}\left(r_{n} / \xi_{\perp}\right)\right]\right\} .
\end{aligned}
$$

The temperature dependence of the correlation length can then in principle be extracted by fitting the measured voltage to Eq. (4.14).

For comparison it is useful to discuss the limiting cases of very small and very large correlation length. Away from the Bose glass transition, where $\xi_{\perp}<<R_{1}, R_{2}, d$, viscous forces are negligible and the boundary condition of vanishing flow velocity at the Bose-glass boundaries cannot be satisfied. The field from vortex motion is simply

$$
e_{r}^{0}=\frac{\rho_{\perp} I}{2 \pi W} \frac{1}{r} .
$$

The voltage drop across each pair of contacts is then given by

$$
\Delta V_{0}\left(r_{n+1}, r_{n}\right)=\frac{\rho_{\perp} I}{2 \pi W} \ln \left(r_{n+1} / r_{n}\right)
$$

and is controlled by the bulk flux flow resistivity $\rho_{\perp}$ given in Eq. (1.8). In this limit the vortex liquid only has very short ranged correlations; the local electric field is simply proportional to the local current and decreases as $\sim 1 / r$. As a result, the voltage across every successive pair of contacts decreases monotonically at all temperatures as one moves from the center to the outer perimeter of the Corbino disk, i.e., $V_{0}\left(r_{2}, r_{1}\right)>V_{0}\left(r_{3}, r_{2}\right)>\ldots>V_{0}\left(r_{n+1}, r_{n}\right)$.

Near the transition, when the correlation length becomes comparable to the channel width, viscous effects are dominant and the field profile can be approximated by

$$
e_{r}^{\infty}(r)=\frac{\rho_{\perp} I}{4 \pi W \xi_{\perp}}\left[b_{1} r-\frac{b_{2}}{r}-r \ln \left(r / \xi_{\perp}\right)\right]
$$

with $b_{1}, b_{2}>0$ given by

$$
\begin{aligned}
& b_{1}=\frac{R_{2}^{2} \ln \left(R_{2} / \xi_{\perp}\right)-R_{1}^{2} \ln \left(R_{1} / \xi_{\perp}\right)}{R_{2}^{2}-R_{1}^{2}}, \\
& b_{2}=\frac{R_{1}^{2} R_{2}^{2}}{R_{2}^{2}-R_{1}^{2}} \ln \left(R_{2} / R_{1}\right) .
\end{aligned}
$$

The voltage drop across two successive contacts is then given by

$$
\begin{aligned}
\Delta V_{\infty}\left(r_{n+1}, r_{n}\right)=\frac{\rho_{\perp} I}{4 \pi W \xi_{\perp}}\{ & \frac{2 b_{1}+1}{4}\left(r_{n+1}^{2}-r_{n}^{2}\right)-b_{2} \ln \left(r_{n+1} / r_{n}\right) \\
& \left.-\frac{1}{2}\left[r_{n+1}^{2} \ln \left(r_{n+1} / \xi_{\perp}\right)-r_{n}^{2} \ln \left(r_{n} / \xi_{\perp}\right)\right]\right\} .
\end{aligned}
$$

The first term on the right hand side of Eq. 4.16 can be easily understood as it corresponds to the field resulting from the rigid body rotation of a vortex solid at an angular frequency $\omega=n_{0} \phi_{0} I /(8 \pi c W \eta)$. In this rigid body limit the electric field grows with $r 33$ As a result, the voltage across two successive contacts increases at all temperatures as one moves from the center to the outer perimeter of the Corbino disk, i.e., $V_{0}\left(r_{2}, r_{1}\right)<V_{0}\left(r_{3}, r_{2}\right)<\ldots<V_{0}\left(r_{n+1}, r_{n}\right)$.

In general as the temperature is decreased towards the Bose glass transition, one will observe a crossover from liquid-like $\left(e_{r} \sim 1 / r\right)$ to solid-like $\left(e_{r} \sim r\right)$ response of the vortex array.

The voltages across each successive pair of five contacts are plotted in Fig. 6 as functions of $d / \xi_{\perp}(T)$. The correlation length $\xi_{\perp}(T)$ increases with temperature as the Bose glass transition is approached from above. The figure therefore displays the growth of the voltage with increasing temperature. At high temperature, where $\xi_{\perp}<<d$, the voltage is given by Eq. (4.15), which is scaled out, and all curves approach 1. The scaled voltage drop across successive pair of contacts is no longer a monotonic function of temperature. Rather it first increases as then decreases as one moves 
from the center towards the outer border of the disk.

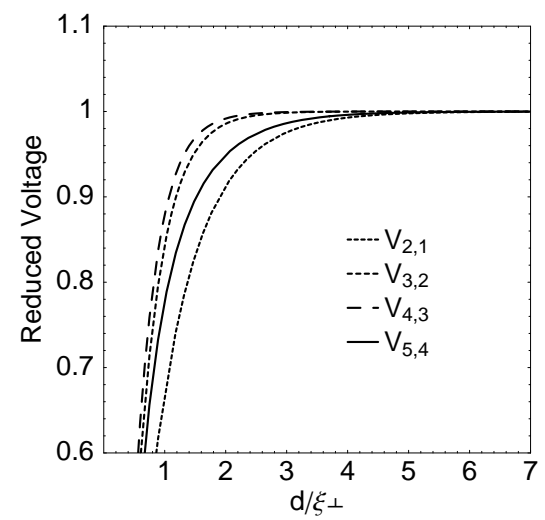

Figure 6. The voltage drop across a series of equally spaced contacts located radially at $r_{n}=R_{1}+n d$, for $n=1,2, \ldots, 5$. The vertical axis represents the voltage across two neighboring contacts scaled by the corresponding voltage obtained for $\xi_{\perp}<<d$, i.e., $V_{n+1, n}=\Delta V\left(r_{n+1}, r_{n}\right) / \Delta V_{0}\left(r_{n+1}, r_{n}\right)$, where $\Delta V\left(r_{n+1}, r_{n}\right)$ and $\Delta V_{0}\left(r_{n+1}, r_{n}\right)$ are given by Eqs. (4.13) and (4.15), respectively. We have chosen $R_{1}=d$ and $R_{2}=6 d$. The voltage is plotted as a function of $d / \xi_{\perp}$ which increases with temperature, as discussed in the text.

\section{RESPONSE AT FINITE FREQUENCY}

The scaling theory of the continuous Bose glass transition in bulk samples is easily generalized to a finite frequency $\omega$, as described in Ref. 17. This is simply done by the addition of another scaling combination $\sim \omega l^{z}$ to the scaling functions of Eqs. (3.17) and (3.18). Similarly, we can generalize to finite frequencies the scaling theory for spatially inhomogeneous flow in constrained geometries. For simplicity we refer again to the channel geometry of Fig. 2 and only discuss the response of the vortex liquid to a spatially homogeneous current of finite frequency $\omega$ applied across the channel in the $a b$ plane. The generalized scaling ansatz based on a renormalization group flow for the spatially inhomogeneous field from flux motion is,

$$
e_{\perp}\left(T, J_{\perp}, \omega, x, L\right)=l^{-(1+z)} e_{\perp}\left(l^{1 / \nu} t, l^{\nu(1+\zeta)} J_{\perp}, l^{z} \omega \tau_{0}, l^{-1} x, l^{-1} L\right)
$$

with $\tau_{0}$ a microscopic time scale. 34 The flow diagram is sketched in Fig. 7 . Clearly the behavior near the critical point depends on how the transition is approached in this plane. For $\omega=0$ we fix the length $l$ as before by choosing $\tilde{t}\left(l_{*}\right)=t l_{*}^{1 / \nu}=1$, which gives $\xi_{\perp}(T) \sim l_{*}=t^{-\nu}$ and yields the temperature dependence discussed earlier for the dc response near the transition. The same analysis should hold at finite, but low frequencies, provided at $l_{*}$ we have $\tilde{t}\left(l_{*}\right)>>\tilde{\omega}\left(l_{*}\right)$, with $\tilde{\omega}(l)=\omega \tau_{0} l^{z}$. This corresponds to the condition $\omega<<1 / \tau(T)$, with $\tau(T) \sim \xi_{\perp}^{z}$ the relaxation time of critical fluctuations. 


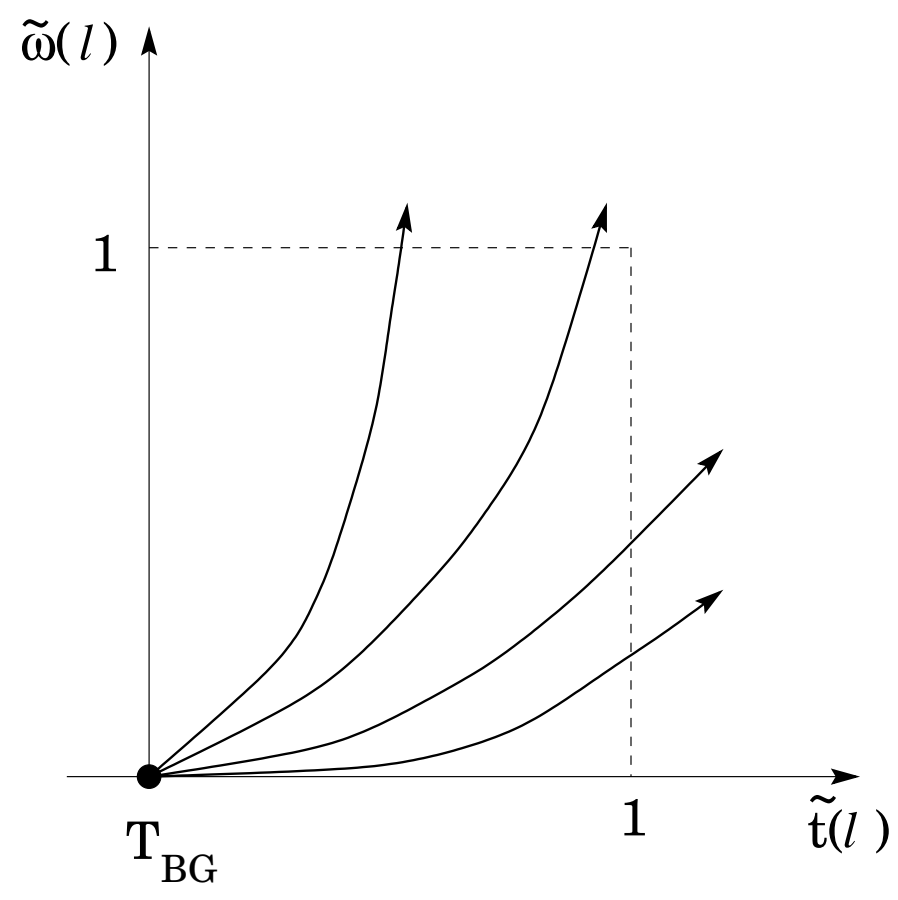

Figure 7. A sketch of the flow diagram in the $(\tilde{\omega}, \tilde{t})$ plane for a few initial values of $\omega \tau_{0} / t^{\nu z}$.

Conversely, we can approach the transition along the frequency axis by letting $T=T_{B G}$ and using a finite frequency to tune the distance from the critical point. In this case we fix $l$ by choosing $\tilde{\omega}\left(l_{*}\right)=1$, which gives $\xi_{\perp}^{\omega} \sim l_{*}=\omega^{-1 / z}$. If the transition is approached along the $T=T_{B G}$ line, the scaling ansatz becomes,

$$
e_{\perp}\left(T=T_{B G}, J_{\perp}, \omega, x, L\right)=\left(\xi_{\perp}^{\omega}\right)^{-(1+z)} \mathcal{E}_{\omega}\left(\left(\xi_{\perp}^{\omega}\right)^{\nu(1+\zeta)} J_{\perp},\left(\xi_{\perp}^{\omega}\right)^{-1} x,\left(\xi_{\perp}^{\omega}\right)^{-1} L\right) .
$$

As before, we are interested in the response in the vortex liquid phase, where the current-voltage characteristic is linear at small currents and we can write

$$
e_{\perp}\left(T=T_{B G}, J_{\perp} \rightarrow 0, \omega, x, L\right)=\rho_{\perp}^{0}\left(\frac{\xi_{\perp}^{\omega}}{a_{0}}\right)^{2-z} J_{\perp} \mathcal{F}_{\omega}\left(x / \xi_{\perp}^{\omega}, L / \xi_{\perp}^{\omega}\right)
$$

By integrating the field across the channel we immediately obtain the net voltage drop and the corresponding ac resistivity as,

$$
\rho_{\perp L}\left(T_{B G}, L, \omega\right)=\rho_{\perp}\left(T_{B G}, \omega\right) f_{\omega}\left(L / \xi_{\perp}^{\omega}\right)
$$

where

$$
\rho_{\perp}\left(T_{B G}, \omega\right)=\rho_{\perp}^{0}\left(\frac{\xi_{\perp}^{\omega}}{a_{0}}\right)^{2-z} \sim(i \omega)^{1-2 / z}
$$

is the ac resistivity in bulk samples. 17 Both real and imaginary parts of the linear frequency-dependent resistivity vanish as $\omega \rightarrow 0$ with the same critical exponent, as obtained earlier in Ref. 17. The scaling function in Eq. (5.3) is simply $f_{\omega}(x)=\frac{1}{x} \int_{-x / 2}^{+x / 2} d u \mathcal{F}_{\omega}(u, x)$. For $x>>1$ the ac resistivity in the channel must reduce to the bulk result, which requires $f_{\omega}(x>>1)=1$.

To determine the form of the scaling function we assume again that at long wavelengths the local field from flux motion is described by the hydrodynamic equations discussed in section II. The use of hydrodynamics for describing the ac response of current-driven vortex liquids has been discussed in Ref. 35. Both friction and viscosity coefficients are in general frequency-dependent. For the simple channel geometry, there is again only one hydrodynamic equation for the Fourier components of the the flow velocity at frequency $\omega$, given by 


$$
-\gamma_{\perp}(\omega) \mathbf{v}+\eta(\omega) \nabla^{2} \mathbf{v}=\frac{1}{c} n_{0} \phi_{0} \hat{\mathbf{z}} \times \mathbf{J}(\omega) .
$$

Determining the frequency dependence of the friction and viscosity coefficients requires a microscopic calculation that is beyond the scope of the present paper. However, their scaling with frequency at the transition can be readily obtained. The hydrodynamic equation (5.6) defines a frequency dependent viscous length, $\sqrt{\eta(\omega) / \gamma_{\perp}(\omega)}$. It is natural to identify this length with the diverging Bose glass correlation length,

$$
\xi_{\perp}^{\omega} \sim \sqrt{\frac{\eta(\omega)}{\gamma_{\perp}(\omega)}} .
$$

The complete scaling function defined by Eq. (5.3) is then readily obtained by solving Eq. (5.6) for no slip boundary conditions and it is found to have the same functional form as that obtained for the dc response. The ac resistivity for the channel geometry if given by

$$
\rho_{\perp L}\left(T_{B G}, L, \omega\right)=\rho_{\perp}\left(T_{B G}, \omega\right)\left[1-\frac{2 \xi_{\perp}^{\omega}}{L} \tanh \left(\frac{L}{2 \xi_{\perp}^{\omega}}\right)\right]
$$

where $\rho_{\perp}\left(T_{B G}, \omega\right)$ is the bulk ac resistivity given in Eq. (5.5). As for the dc case, one obtains the following behavior,

$$
\begin{aligned}
& \rho_{\perp L}\left(T_{B G}, L, \omega\right) \approx\left(\frac{n_{0} \phi_{0}}{c}\right)^{2} \frac{1}{\gamma_{\perp}(\omega)} \sim(i \omega)^{1-2 / z}, \quad \text { for } \quad \xi_{\perp}^{\omega}<<L, \\
& \rho_{\perp L}\left(T_{B G}, L, \omega\right) \approx\left(\frac{n_{0} \phi_{0}}{c}\right)^{2} \frac{L^{2}}{12 \eta(\omega)} \sim L^{2}\left(\xi_{\perp}^{\omega}\right)^{-z} \sim L^{2}(i \omega), \quad \text { for } \quad \xi_{\perp}^{\omega}<<L .
\end{aligned}
$$

The condition $\xi_{\perp}^{\omega} \sim L$ defines a sample-dependent crossover frequency $\omega_{L}=\left(a_{0} / L\right)^{z} / \tau_{0} \sim L^{-z}$ below which the channel geometry becomes important and $\rho_{\perp L} \sim i \omega L^{2}$. These results describe a Meissner-type response of the superconductor that arises because the vortices are pinned and immobile in the Bose glass phase.

The behavior observed as the critical point is approached along an arbitrary line in the $(\omega, t)$ plane depends on the location of that line. In general we expect that the $\omega=0$ scaling results apply for $\omega<<1 / \tau(T) \sim t^{z}$, while the scaling for $T=T_{B G}$ will apply for $\omega>>1 / \tau$. The various regions for the expected scaling of the ac response are sketched in Fig. 8. Here the temperature scale $t_{L}$ is defined by $\xi_{\perp}(T) \sim L$, with $t_{L} \sim\left(a_{0} / L\right)^{1 / \nu}$. For $t>t_{L}$ the vortex array is insensitive to the confined geometry and the response is that of a bulk sample. For temperatures below $t_{L}$, the geometry becomes important.

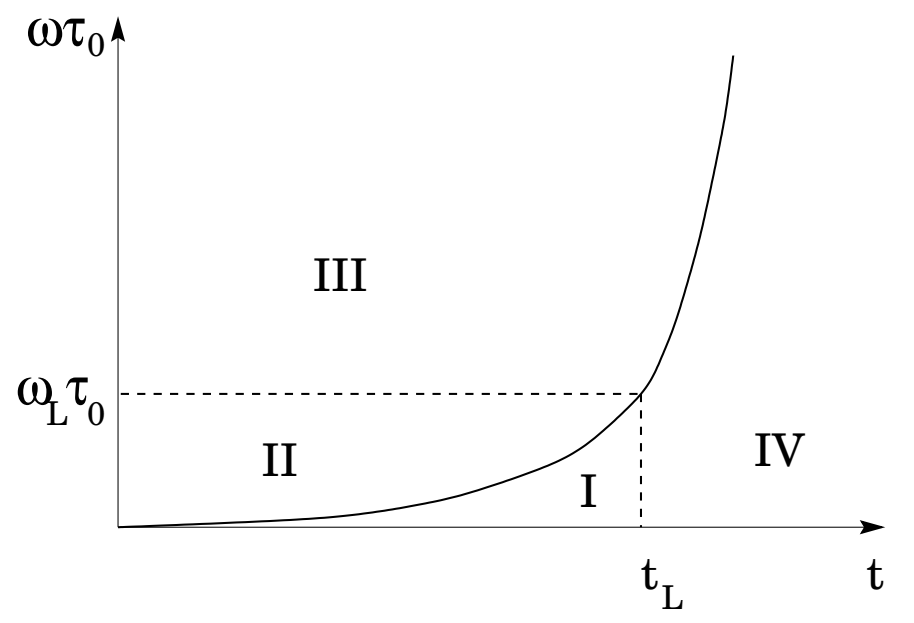

Figure 8. A sketch of the scaling behavior expected for the ac response in various regions of the $(\omega, t)$ plane. The solid line is a plot of $\omega=1 / \tau(T)=t^{z \nu} / \tau_{0}$. The frequency and temperature scales $\omega_{L}$ and $t_{L}$ represent the scales below which the confined sample geometry becomes important. They are defined by $\xi_{\perp}^{\omega_{L}}=L$, or $\omega_{L} \tau_{0}=\left(a_{0} / L\right)^{z}$, and by $\xi_{\perp}\left(T_{L}\right)=L$, which gives $t_{L}=\left(a_{0} / L\right)^{1 / \nu}$. The ac resistivity $\rho_{\perp L}(\omega, T)$ is expected to scale as follows deep in each 
of the four regions sketched in the figure: region I, $\rho_{\perp L} \sim L^{2} t^{\nu z}$; region II, $\rho_{\perp L} \sim L^{2}(i \omega) ;$ region III, $\rho_{\perp L} \sim(i \omega)^{1-2 / z}$; region IV, $\rho_{\perp L} \sim t^{\nu(z-2)}$.

This work was supported by the National Science Foundation at Syracuse through Grants No. DMR97-30678 and DMR98-05818 and at Harvard through Grant No. DMR97-14725, and by the Harvard Materials Research Science and Engineering Center through Grant No. DMR98-09363.

\section{APPENDIX A: COMPARISON WITH THE HYDRODYNAMIC FORMULATION OF MOU $E T A L$.}

Mou et al. have used time-dependent Ginzburg-Landau theory to evaluate the nonlocal dc conductivity tensor $\Sigma_{\mu \nu}\left(\mathbf{r}, \mathbf{r}^{\prime}\right)$ defined in Eq. (2.5). Assuming nonlocal effects are slowly varying on the length scale of interest, one can expand the right hand side of Eq. (2.5) retaining terms up to quadratic in the gradients of the electric field. At long wavelengths, the nonlocal relation between fields and currents then takes the form,

$$
j_{\mu}(\mathbf{r})=\sigma_{\mu \nu}(\mathbf{0}) e_{\nu}(\mathbf{r})-S_{\mu \alpha \beta \nu} \partial_{\alpha} \partial_{\beta} e_{\nu}(\mathbf{r})
$$

where $\sigma_{\mu \nu}(\mathbf{0})$ is the usual long-wavelength conductivity and the tensor $S_{\mu \alpha \beta \nu}$ describes the effect of nonlocality to quadratic order in the gradients. This fourth order tensor has six independent components that were evaluated in Ref. 28.

Upon comparing Eq. (A1) to Eq. (2.30) and recalling the relationship between the fields and the vortex densities and fluxes given by the long-wavelength limit of Eqs. (2.11) and (2.13),

$$
\begin{aligned}
& \mathbf{b}=\phi_{0} \mathbf{T}, \\
& e_{\mu}=\frac{\phi_{0}}{c} \epsilon_{\mu \nu \mathrm{J}} Q_{\nu j},
\end{aligned}
$$

we see immediately that the local conductivity tensor $\sigma_{\mu \nu}(\mathbf{0})$ and the tensor $S_{\mu \alpha \beta \nu}$ are simply proportional to the friction and viscosity tensor, respectively, according to,

$$
\begin{aligned}
& \sigma_{\gamma \sigma}(\mathbf{0})=\frac{c^{2}}{\phi_{0}^{2}} \epsilon_{\gamma \mu \nu} \tilde{\gamma}_{\mu \nu, \lambda \rho} \epsilon_{\lambda \rho \sigma}, \\
& S_{\gamma \alpha \beta \sigma}=\frac{c^{2}}{\phi_{0}^{2}} \epsilon_{\gamma \mu \nu} \tilde{\eta}_{\mu \nu, \alpha \beta, \lambda \rho} \epsilon_{\lambda \rho \sigma} .
\end{aligned}
$$

Neglecting Hall voltages, the local conductivity tensor is diagonal and has only two independent components, due to the rotational symmetry in the plane normal to the applied field $(x y$ plane $), \sigma_{x x}(\mathbf{0})=\sigma_{y y}(\mathbf{0})$ and $\sigma_{z z}(\mathbf{0})$, which gives

$$
\sigma_{\alpha \beta}(\mathbf{0})=\sigma_{x x}(\mathbf{0}) \delta_{\alpha \beta}^{\perp}+\sigma_{z z}(\mathbf{0}) \delta_{\alpha z} \delta_{\beta z}
$$

where $\delta_{\alpha \beta}^{\perp}=\delta_{\alpha_{\perp}, \beta_{\perp}}=\delta_{\alpha \beta}-\delta_{\alpha z} \delta_{\beta z}$ and the labels with a $\perp$ subscript, e.g., $\alpha_{\perp}, \beta_{\perp}, \ldots$, only run over the two values $x$ and $y$. The friction tensor is given by

$$
\tilde{\gamma}_{\mu \nu, \lambda \rho}=\frac{1}{2 n_{0}^{2}}\left(\gamma_{\perp} \epsilon_{\mu \nu \alpha_{\perp}} \epsilon_{\alpha_{\perp} \lambda \rho}+\gamma_{\|} \epsilon_{\mu \nu z} \epsilon_{\alpha \lambda z}\right),
$$

with

$$
\begin{aligned}
\sigma_{x x}(\mathbf{0}) & =\left(\frac{c}{n_{0} \phi_{0}}\right)^{2} \gamma_{\perp} \\
\sigma_{z z}(\mathbf{0}) & =\left(\frac{c}{n_{0} \phi_{0}}\right)^{2} \gamma_{\|}
\end{aligned}
$$

Symmetry under rotations about the $z$ direction also restricts the number of independent components of the viscosity tensor and the related tensor $S_{\mu \alpha \beta \nu}$ to six. The tensor $S_{\mu \alpha \beta \nu}$ is given by 


$$
\begin{aligned}
S_{\mu \alpha \beta \nu}= & S_{x x x x} \delta_{\mu \nu}^{\perp} \delta_{\alpha \beta}^{\perp}+\left(S_{x y y x}-S_{x x x x}\right)\left[\delta_{\mu \nu}^{\perp} \delta_{\alpha \beta}^{\perp}-\frac{1}{2}\left(\delta_{\mu \alpha}^{\perp} \delta_{\beta \nu}^{\perp}+\delta_{\mu \beta}^{\perp} \delta_{\alpha \nu}^{\perp}\right)\right] \\
& +S_{x z z x} \delta_{\mu \nu}^{\perp} \delta^{\alpha z} \delta^{\beta z}+S_{z x x z} \delta_{\alpha \beta}^{\perp} \delta^{\mu z} \delta^{\nu z}+S_{z z z z} \delta_{\mu z} \delta_{\alpha z} \delta_{\beta z} \delta_{\nu z} \\
& +S_{x x z z}\left(\delta_{\alpha z} \delta_{\mu z} \delta_{\beta \nu}^{\perp}+\delta_{\beta z} \delta_{\nu z} \delta_{\alpha \mu}^{\perp}+\delta_{\beta z} \delta_{\mu z} \delta_{\alpha \nu}^{\perp}+\delta_{\alpha z} \delta_{\nu z} \delta_{\beta \mu}^{\perp}\right) .
\end{aligned}
$$

The viscosity tensor is given by

$$
\begin{aligned}
\tilde{\eta}_{\mu \nu, \alpha \beta, \lambda \rho}=\frac{1}{2 n_{0}^{2}}\{ & \eta \delta_{\alpha \beta}^{\perp} \epsilon_{\mu \nu \gamma_{\perp}} \epsilon_{\gamma_{\perp} \lambda \rho}+\eta_{b}\left[\delta_{\alpha \beta}^{\perp} \epsilon_{\mu \nu \gamma_{\perp}} \epsilon_{\gamma_{\perp} \lambda \rho}-\frac{1}{2}\left(\epsilon_{\mu \nu \alpha_{\perp}} \epsilon_{\beta_{\perp} \lambda \rho}+\epsilon_{\mu \nu \beta_{\perp}} \epsilon_{\alpha_{\perp} \lambda \rho}\right)\right] \\
& +\eta_{z} \delta_{\alpha z} \delta_{\beta z} \epsilon_{\mu \nu \gamma_{\perp}} \epsilon_{\gamma_{\perp} \lambda \rho}+\eta^{t} \delta_{\alpha \beta}^{\perp} \epsilon_{\mu \nu z} \epsilon_{z \lambda \rho}+\eta_{z}^{t} \delta_{\alpha z} \delta_{\beta z} \epsilon_{\mu \nu z} \epsilon_{z \lambda \rho} \\
& \left.+\frac{1}{2} \eta_{x}\left[\delta_{\alpha z}\left(\epsilon_{\mu \nu z} \epsilon_{\beta_{\perp} \lambda \rho}+\epsilon_{\mu \nu \beta_{\perp}} \epsilon_{z \lambda \rho}\right)+\delta_{\beta z}\left(\epsilon_{\mu \nu z} \epsilon_{\alpha_{\perp} \lambda \rho}+\epsilon_{\mu \nu \alpha_{\perp}} \epsilon_{z \lambda \rho}\right)\right]\right\}
\end{aligned}
$$

with

$$
\begin{aligned}
& S_{x x x x}=\left(\frac{c}{n_{0} \phi_{0}}\right)^{2} \eta, \\
& S_{x z z x}=\left(\frac{c}{n_{0} \phi_{0}}\right)^{2} \eta_{z}, \\
& \left(S_{x y y x}-S_{x x x x}\right)=\left(\frac{c}{n_{0} \phi_{0}}\right)^{2} \eta_{b}, \\
& S_{z x x z}=\left(\frac{c}{n_{0} \phi_{0}}\right)^{2} \eta^{t}, \\
& S_{z z z z}=\left(\frac{c}{n_{0} \phi_{0}}\right)^{2} \eta_{z}^{t}, \\
& S_{x x z z}=\frac{1}{2}\left(\frac{c}{n_{0} \phi_{0}}\right)^{2} \eta_{x} .
\end{aligned}
$$

Mou et al. evaluated the lowest order fluctuation contribution to the components of the tensor $S_{\mu \alpha \beta \nu}$ for a vortex liquid using a time-dependent Ginzburg-Landau theory within a Gaussian approximation.

\section{APPENDIX B: DC FLUX TRANSFORMER EXPERIMENTS}

DC flux transformer experiments have been used as probes of the nonlocal resistive response of vortex liquids. In particular, by comparing transport measurements in twinned and untwinned $Y B C O$, Lopéz et al 36 argued that in the twinned crystal, where the liquid transforms into a solid via a continuous glass transition, measurements in the transformer configuration may provide a direct probe of the longitudinal correlation length near the transition. Here we reconsider the analysis of such experiments in the context of hydrodynamics following earlier work by Huse and Majumdar.32. Our objective is to make the connection between the measured voltages and the diverging correlation lengths more precise. As pointed out by Huse and Majumdar, explicit calculations of the current and voltage patterns have to be carried out for each specific sample geometry by solving a fourth order partial differential equation with rather complicated boundary conditions. Here we reformulate the problem discussed in Ref. 32 incorporating all relevant viscosity coefficients, then discuss the solution in terms of diverging length scales. We also correct Eq. (7) of Ref. 32 where some boundary terms were left out.

We are interested in discussing dc transport experiments in finite materials of specified geometry, where the current is injected and withdrawn via contacts placed at the materials' boundaries. As discussed by Huse and Majumdar, in the bulk of the sample in steady state the total current satisfies $\boldsymbol{\nabla} \cdot \mathbf{j}=0$ and the electric field is irrotational, $\boldsymbol{\nabla} \times \mathbf{e}=0$. The relationship between current and field is given by the nonlocal Ohm's law, Eq. (2.5), which at long wavelength takes the form given in Eq. (A1). The field e can be written in terms of a local voltage as

$$
\mathbf{e}=-\nabla \Phi
$$

and the nonlocal Ohm's law transforms into a fourth order differential equation for the voltage, $\Phi$,

$$
\left[\sigma_{\mu \nu}(\mathbf{0}) \partial_{\mu} \partial_{\nu}-S_{\mu \alpha \beta \nu} \partial_{\mu} \partial_{\alpha} \partial_{\beta} \partial_{\nu}\right] \Phi=0
$$


The sample boundaries separate a region where the local field or vortex flow velocity can have spatial gradients due to viscous forces among the vortices from the outside region where no such spatial gradients occur. Neglecting nonlocalities on length scales of the order of the penetration length, the first spatial derivatives of the field $\mathbf{e}$ (or equivalently of the vortex flux tensor $Q_{\mu \nu}$ ) can therefore exhibit jump discontinuities at the sample boundaries. Such discontinuities correspond to $\delta$-function contributions to the local current, denoted by $\mathbf{j}^{\delta}$. Considering a local coordinate system with an $s$ axis normal to the sample boundary, located at $s=0$, and directed inwards, the nonlocal Ohm's law at the boundary takes the form,

$$
j_{\mu}(\mathbf{r})+j_{\mu}^{\delta}(\mathbf{r})=\sigma_{\mu \nu}(\mathbf{0}) e_{\nu}(\mathbf{r})-S_{\mu \alpha \beta \nu} \partial_{\alpha} \partial_{\beta} e_{\nu}(\mathbf{r})-\delta(s) S_{\mu s s \nu} \partial_{\nu}\left[e_{\nu}(\mathbf{r})\right]_{s \rightarrow 0^{+}},
$$

where the subscript $s$ is not summed over. The last term on the right hand side of the equation equals the $\delta$ function contribution to the surface current. Such contribution must be flowing within the surface of the sample, i.e., its component normal to the boundary must vanish,

$$
j_{s}^{\delta}=S_{s s s \nu}\left[\partial_{s} e_{\nu}\right]_{s \rightarrow 0^{+}}=-S_{s s s \nu}\left[\partial_{s} \partial_{\nu} \Phi\right]_{s \rightarrow 0^{+}}=0 .
$$

This provides the first set of boundary conditions for Eq. (B2) The second boundary condition is obtained by requiring that the $\delta$-function part of the divergence of the current at the surface equals the total current $I_{s}(s)$ injected at that point of the surface,

$$
(\boldsymbol{\nabla} \cdot \mathbf{j})^{\delta}=\boldsymbol{\nabla} \cdot \mathbf{j}^{\delta}=-\delta(s) S_{\mu_{\perp} s s \nu} \partial_{\mu_{\perp}} \partial_{\nu}\left[e_{\nu}(\mathbf{r})\right]_{s \rightarrow 0^{+}}=I_{s}(s),
$$

where again the index $s$ is not summed over and $\mu_{\perp}$ only runs over the two components perpendicular to the surface. This requires that the voltage at the surface satisfies,

$$
\left[\sigma_{s \nu}(\mathbf{0}) \partial_{\nu} \Phi-S_{s \alpha \beta \nu} \partial_{\mu} \partial_{\alpha} \partial_{\beta} \partial_{\nu} \Phi-S_{\mu_{\perp} s \nu} \partial_{\mu_{\perp}} \partial_{s} \partial_{\nu} \Phi-2 S_{\mu_{\perp} \nu s s} \partial_{\mu_{\perp}} \partial_{s} \partial_{\nu} \Phi\right]_{s=0^{+}}=I_{s}(s) .
$$

The last term in square brackets on the left hand side of Eq. (B6) was absent in Ref. 32.

The specific transformer configurations of interest are shown in Fig. 9. We neglect spatial inhomogeneities in the $x$ direction (parallel to the flow) and reduce the problem to a two-dimensional one. The sample has finite extent $W$ in 
the $y$ direction and thickness $L$ in the external field direction.

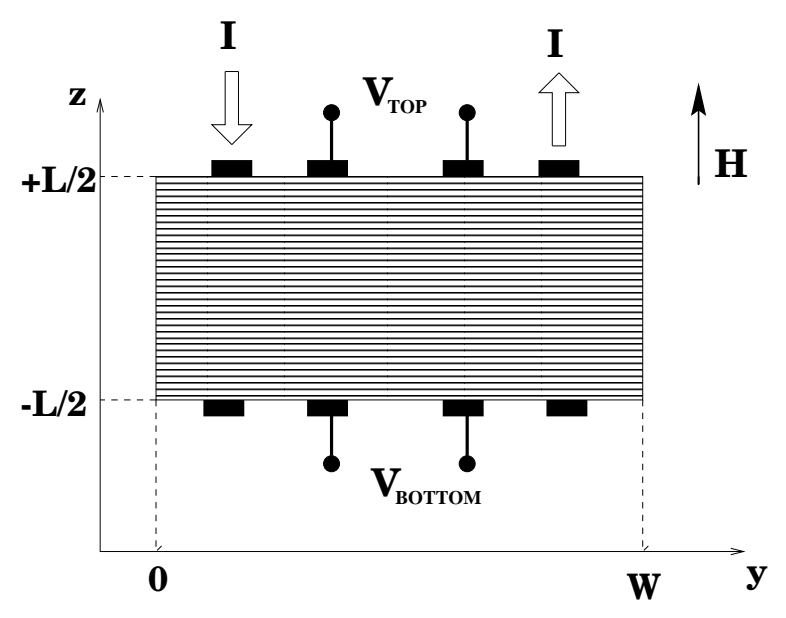

(a)

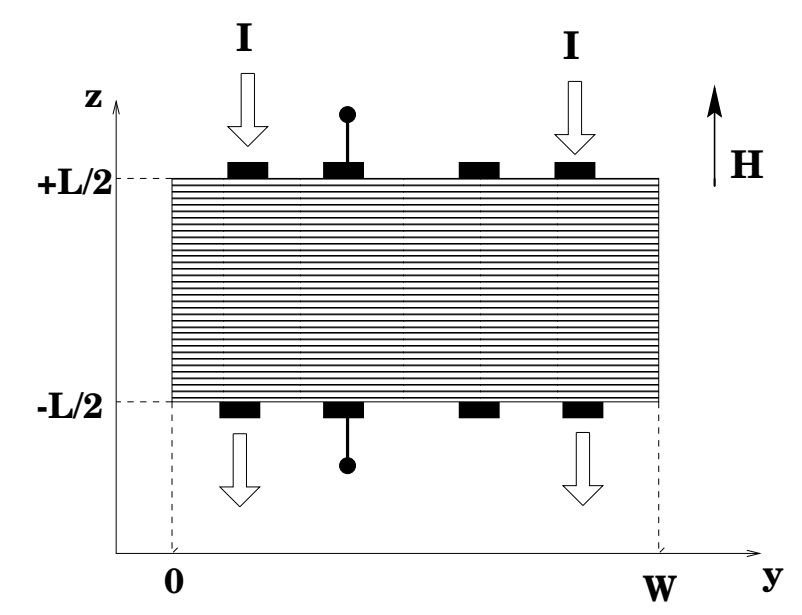

(b)

Figure 9. The dc flux transformer geometry.

Current is injected and withdrawn at the contacts shown placed on the upper and lower surface of the sample. Therefore no current is injected at the boundaries normal to the $y$ direction. In order to discuss the role of the various viscosity coefficients in controlling the nonlocal response of the vortex array, it is useful to return to our hydrodynamic equations in terms of vortex densities and fluxes. In the geometry of interest there are two nonvanishing components of the electric field from flux motion, related to the corresponding components of the vortex flux tensor as

$$
\begin{aligned}
& e_{y}(y, z)=\frac{n_{0} \phi_{0}}{c} v_{x}(y, z), \\
& e_{z}(y, z)=\frac{n_{0} \phi_{0}}{c} V(y, z) .
\end{aligned}
$$

From Eq. (2.15) in a steady state vortex current is conserved, which requires

$$
\partial_{z} V=\partial_{y} v_{x},
$$

This condition is equivalent to the requirement that $\boldsymbol{\nabla} \times \mathbf{e}=0$ and implies that the components of the vortex flux tensor can be derived by a potential, $\Phi_{v}$, according to 


$$
\begin{aligned}
& v_{x}(y, z)=\partial_{y} \Phi_{v}, \\
& V(y, z)=\partial_{z} \Phi_{v},
\end{aligned}
$$

where $\Phi_{v}$ is simply proportional to the voltage defined in Eq. (B1), $\Phi_{v}=-\frac{c}{n_{0} \phi_{0}} \Phi$. The hydrodynamic equations governing vortex flow in the transformer geometry shown in Fig. 9 are

$$
\begin{aligned}
& \gamma_{\perp} v_{x}=\left(\eta \partial_{y}^{2} \eta_{z} \partial_{z}^{2}\right) v_{x}+\eta_{x} \partial_{y} \partial_{z} V+\frac{c_{44}}{n_{0}} \partial_{z} t_{x}+\frac{n_{0} \phi_{0}}{c} J_{y}, \\
& \gamma_{\|} V=\left(\eta^{t} \partial_{y}^{2}+\eta_{z}^{t} \partial_{z}^{2}\right) V+\eta+x \partial_{y} \partial_{z} v_{x}-\frac{c_{44}}{n_{0}} \partial_{y} t_{x}+\frac{n_{0} \phi_{0}}{c} J_{z} .
\end{aligned}
$$

By differentiating Eq. (B10) with respect to $y$ and Eq. (B11) with respect to $z$ and making use again of the fact that the total current is divergence-free in bulk, we immediately obtain the equation for the potential $\Phi_{v}$,

$$
\gamma_{\perp} \partial_{y}^{2} \Phi_{v}+\gamma_{\|} \partial_{z}^{2} \Phi_{v}-\left[\eta \partial_{y}^{4}+\left(\eta_{z}+\eta^{t}+2 \eta_{x}\right) \partial_{y}^{2} \partial_{z}^{2}+\eta_{z}^{t} \partial_{z}^{4}\right] \Phi_{v}=0
$$

This fourth order differential equation has to be solved with the boundary conditions,

$$
\begin{aligned}
& \eta\left[\partial_{y}^{2} \Phi_{v}\right]_{y=0, W}=0, \\
& \eta_{z}^{t}\left[\partial_{z}^{2} \Phi_{v}\right]_{z=0, L}=0, \\
& {\left[\gamma_{\perp} \partial_{y} \Phi_{v}-\eta \partial_{y}^{3} \Phi_{v}-\left(\eta_{z}+2 \eta_{x}+\eta^{t}\right) \partial_{z}^{2} \partial_{y} \Phi_{v}\right]_{y=0, W}=0,} \\
& {\left[\gamma_{\|} \partial_{z} \Phi_{v}-\eta_{z}^{t} \partial_{z}^{3} \Phi_{v}-\left(\eta_{z}+2 \eta_{x}+\eta^{t}\right) \partial_{y}^{2} \partial_{z} \Phi_{v}\right]_{z=0, L}= \pm \frac{c}{n_{0} \phi_{0}} I_{z}\left(y, z= \pm \frac{L}{2}\right) .}
\end{aligned}
$$

To proceed, we follow Huse and Majumdar and neglect viscous drag associated with $\eta$ and $\eta_{z}^{t}$ (the bulk viscosity $\eta_{b}$ drops out of the problem for linear incompressible flow). This amounts to dropping the terms $\sim \partial_{y}^{4}$ and $\sim \partial_{z}^{4}$ compared to the fourth order cross derivatives in Eq. (B12). Letting $\tilde{\eta}_{z}=\eta_{z}+\eta^{t}+2 \eta_{x} \mathrm{BT}$, the equation simply reduces to

$$
\gamma_{\perp} \partial_{y}^{2} \Phi_{v}+\gamma_{\|} \partial_{z}^{2} \Phi_{v}-\tilde{\eta}_{z} \partial_{y}^{2} \partial_{z}^{2} \Phi_{v}=0
$$

with the boundary conditions

$$
\begin{aligned}
& {\left[\gamma_{\perp} \partial_{y} \Phi_{v}-\tilde{\eta}_{z} \partial_{z}^{2} \partial_{y} \Phi_{v}\right]_{y=0, W}=0} \\
& {\left[\gamma_{\|} \partial_{z} \Phi_{v}-\tilde{\eta}_{z} \partial_{y}^{2} \partial_{z} \Phi_{v}\right]_{z=0, L}= \pm \frac{c}{n_{0} \phi_{0}} I_{z}(y, \pm L / 2) .}
\end{aligned}
$$

The solution for an arbitrary distribution of current sources and sinks has been given by Huse and Majumdar. 32 Simply translating their results into our notation, we write the current sources at the top and bottom of the sample as

$$
I(y, z= \pm L / 2)=\sum_{n=0}^{\infty} i_{n}^{ \pm} \cos (n \pi y / W)
$$

The integral of the current injected or withdrawn over the entire sample surface must vanish. This requires $i_{0}^{+}=-i_{0}^{-}$. Making use of the boundary conditions at $x=0, W$, the voltage is then written as

$$
\Phi(y, z)=\sum_{n=0}^{\infty} U_{n}(z) \cos (n \pi y / W)
$$

where

$$
\begin{aligned}
& U_{0}(z)=C_{0}+S_{0} z, \\
& U_{n}(z)=C_{n} \cosh \left(K_{n} z\right)+S_{n} \sinh \left(K_{n} z\right), \quad n>0,
\end{aligned}
$$


with

$$
K_{n}=\frac{n \pi}{W}\left(\frac{\gamma_{\perp}(T)}{\gamma_{\|}(T)}\right)^{1 / 2}\left[1+\frac{\tilde{\eta}_{z}(T)}{\gamma_{\|}(T)}(n \pi / W)^{2}\right]^{-1 / 2} .
$$

The coefficient $C_{0}$ sets an arbitrary zero for the voltage. We will let $C_{0}=0$ below. The other coefficients are given by

$$
\begin{aligned}
& S_{0}=\rho_{\|} i_{0}^{-}, \\
& S_{n}=\frac{\rho_{\perp}\left(i_{n}^{-}-i_{n}^{+}\right)}{2} \frac{K_{n}(W / n \pi)^{2}}{\cosh \left(K_{n} L / 2\right)}, \quad n>0, \\
& C_{n}=-\frac{\rho_{\perp}\left(i_{n}^{-}+i_{n}^{+}\right)}{2} \frac{K_{n}(W / n \pi)^{2}}{\sinh \left(K_{n} L / 2\right)}, \quad n>0 .
\end{aligned}
$$

Now we use this solution to discuss the experimental finding of Lopeź et al 36 in twinned and untwinned samples of $Y B C O$. These authors have carried out two sets of measurements. The first consists in injecting current at the left contact at the top of the sample and extracting it at the right contact, as shown in Fig. 9. The voltages $V_{\text {TOP }}$ and $V_{\text {Воттом }}$ across the two central contacts at the top and bottom of the sample are then measured as functions of temperature. For this choice of current sources, $i_{n}^{-}=0$, for all $n$, and $i_{0}^{+}=0$. The voltage at the top and bottom of the sample is then given by

$$
\Phi(y, \pm L / 2)=-\frac{1}{2} \rho_{\perp} \sum_{n=1}^{\infty} i_{n}^{+} \cos (n \pi y / W) K_{n}\left(\frac{W}{n \pi}\right)^{2}\left[\frac{\cosh \left(K_{n} L / 2\right)}{\sinh \left(K_{n} L / 2\right)} \pm \frac{\sinh \left(K_{n} L / 2\right)}{\cosh \left(K_{n} L / 2\right)}\right] .
$$

The temperature dependence of the voltages is controlled by that of the inverse length $K_{n}$, given in Eq. (B19), which in turn depends on the friction and viscosity coefficients which diverge near the vortex glass and the Bose glass transitions with the critical exponents discussed earlier. In particular we recall that

$$
\begin{aligned}
& \frac{\gamma_{\perp}(T)}{\gamma_{\|}(T)}=\frac{\gamma_{\perp}^{0}}{\gamma_{\|}^{0}}\left(\frac{\xi_{\perp}}{\xi_{\|}}\right)^{2} \sim t^{2 \nu(\zeta-1)}, \\
& \frac{\tilde{\eta}_{z}(T)}{\gamma_{\|}(T)}=\frac{\tilde{\eta}_{z}^{0}}{\gamma_{\|}^{0}}\left(\frac{\xi_{\perp}}{a_{0}}\right)^{2} \sim t^{2 \nu},
\end{aligned}
$$

where we have extracted the divergence by rewriting all transport properties in terms of their bare values (denoted by a superscript "0"), which depend only weakly on temperature at the transition. The longitudinal correlation length will be defined as

$$
\xi_{\|}^{2}=\tilde{\eta}_{z}(T) / \gamma_{\perp}(T)
$$

We begin by discussing the case of vanishing viscosity. Then $K_{n}$ is simply given by

$$
K_{n} \approx K_{n}^{0}=\frac{n \pi}{W} \sqrt{\frac{\gamma_{\perp}}{\gamma_{\|}}}=\frac{n \pi}{W} \sqrt{\frac{\rho_{\|}}{\rho_{\perp}}} \sim t^{2 \nu(\zeta-1)} .
$$

It vanishes therefore at the Bose glass transition $(\zeta=2)$, but it remains finite at the isotropic vortex glass transition $(\zeta=1)$. Choosing for simplicity a current distribution with $i_{1}^{+} \neq 0$ and $i_{n}^{+}=0$ for all other values of $n$, the voltage for $\tilde{\eta}_{z}=0$ is given by

$$
\Phi_{0}(y, \pm L / 2)=-\frac{1}{2} \sqrt{\rho_{\|} \rho_{\perp}} i_{1}^{+} \cos (\pi y / W)\left(\frac{W}{\pi}\right)^{2}\left[\frac{\cosh \left(K_{1}^{0} L / 2\right)}{\sinh \left(K_{1}^{0} L / 2\right)} \pm \frac{\sinh \left(K_{1}^{0} L / 2\right)}{\cosh \left(K_{1}^{0} L / 2\right)}\right]
$$

with $K_{1}^{0}$ given by Eq. B25 for $n=1$. Close to the glass transition where $K_{1}^{0} \sim t^{2 \nu(\zeta-1)}$ we can use $K_{1}^{0} L<<1$ to obtain,

$$
\Phi_{0}(y, \pm L / 2) \approx-\rho_{\perp} \frac{W^{2}}{\pi^{2} L} i_{1}^{+} \cos (\pi y / W),
$$

and the voltage at the top and bottom of the sample are equal. Conversely, if $K_{1}^{0} L>>1$, we find 


$$
\begin{aligned}
& \Phi_{0}(y,+L / 2) \approx-\sqrt{\rho_{\|} \rho_{\perp}} \frac{W}{\pi} i_{1}^{+} \cos (\pi y / W), \\
& \Phi_{0}(y,-L / 2) \approx 0,
\end{aligned}
$$

with corrections to the above expressions decreasing exponentially as $\sim e^{-K_{1}^{0} L}$. In this case the vortices behave like uncorrelated pancakes and the bottom voltage is much smaller than the one at the top. In other words, there is a crossover at $K_{1}^{0} L \sim 1$, corresponding to $\rho_{\|} L^{2} / 4 \sim \rho_{\perp} W^{2} / \pi^{2}$, from a regime where the top voltage exceeds the bottom voltage to one where both voltages are the same. Near the Bose glass transition, where $K_{1}^{0} \sim t^{2 \nu}$, this crossover is sharp and occurs at a temperature that depends on both the width $W$ and the thickness Lof the sample and is given by $t_{*}^{\nu} \sim(2 W / \pi L) \sqrt{\gamma_{\|}^{0} / \gamma_{\perp}^{0}}$. In contrast, near the vortex glass transition where $\zeta=1, K_{1}^{0}$ is weakly temperature dependent and no sharp crossover is expected.

Consider now the response in the case where the viscosity $\tilde{\eta}_{z}$ is very large, with $\left(\tilde{\eta}_{z} / \gamma_{\|}\right)(\pi / W)^{2}>>1$, so that for all values of $n$,

$$
K_{n} \approx K_{n}^{\infty}=\sqrt{\frac{\gamma_{\perp}}{\tilde{\eta}_{z}}}=\frac{1}{\xi_{\|}}
$$

In this case the top and bottom voltages for the first experiment (Fig. 9) are given by

$$
\Phi_{\infty}(y, \pm L / 2) \approx-\frac{1}{2} \frac{\rho_{\perp}}{\xi_{\|}}\left[\frac{\cosh \left(L / 2 \xi_{\|}\right)}{\sinh \left(L / 2 \xi_{\|}\right)} \pm \frac{\sinh \left(L / 2 \xi_{\|}\right)}{\cosh \left(L / 2 \xi_{\|}\right)}\right] \sum_{n=1}^{\infty} i_{n}^{+} \cos (n \pi y / W)\left(\frac{W}{n \pi}\right)^{2} .
$$

The crossover is now controlled by the length $\xi_{\|} \sim t^{-\zeta \nu}$. If $L / 2 \xi_{\|}>>1$, the voltage reduces to

$$
\begin{aligned}
& \Phi_{\infty}(y,+L / 2) \approx-\frac{\rho_{\perp}}{\xi_{\|}} \sum_{n=1}^{\infty} i_{n}^{+} \cos (n \pi y / W)\left(\frac{W}{n \pi}\right)^{2}, \\
& \Phi_{\infty}(y,-L / 2) \approx 0,
\end{aligned}
$$

i.e., voltages are uncorrelated well above the glass transition. As the glass transition is approached and $L / 2 \xi_{\|}<<1$, we obtain,

$$
\Phi_{\infty}(y, \pm L / 2) \approx-\frac{\rho_{\perp}}{L} \sum_{n=1}^{\infty} i_{n}^{+} \cos (n \pi y / W)\left(\frac{W}{n \pi}\right)^{2} .
$$

In this case the top and bottom voltages are the same and they are both reduced by a factor $\xi_{\|} / L$ compared to the top voltage at high temperature. Their vanishing at the transition is controlled by the vanishing of $\rho_{\perp}$. We find a sharp crossover from a high temperature region where the top voltage exceeds the bottom voltage to a low temperature region where the two voltages are equal. the crossover temperature $T^{*}$ depends on the sample thickness and is given by $\xi_{\|}\left(T^{*}\right) \sim L / 2$. It is therefore expected to decrease with sample thickness according to $T^{*} \sim T_{B G}\left(L / a_{0}\right)^{-1 / \zeta \nu}$, consistent with the observations of Lopeź et al. in twinned samples. For $T<T^{*}$ the voltages are the same and decreasing with decreasing temperature, vanishing at $T_{B G}$ as $\rho_{\perp} \sim t^{\nu(z-\zeta)}$.

The second set of measurements carried out by Lopeź et al. consists of injecting a current at the top surface and extracting it at the bottom surface, while measuring the voltage across two contacts at $z= \pm L / 2$ and at the same $y$ position, as sketched in Fig. 9 . The corresponding distribution of current sources and sinks corresponds to $i_{n}^{+}=-i_{n}^{-}$, for every $n$. The voltage difference between top and bottom is given by

$$
\begin{aligned}
\Delta \Phi(y) & \equiv \Phi(y, L / 2)-\Phi(y,-L / 2) \\
& =i_{0}^{-} \rho_{\|} L+2 \rho_{\perp} \sum_{n=1}^{\infty} i_{n}^{-} \cos (n \pi y / W) K_{n}\left(\frac{W}{n \pi}\right)^{2} \tanh \left(K_{n} L / 2\right) .
\end{aligned}
$$

For simplicity we assume the currents injected at the top and withdrawn at the bottom are uniform in $y$, i.e., $i_{0}^{-}=-i_{0}^{+}=$constant, and $i_{n}^{-}=-i_{n}^{+}=0$ for $n \neq 1$. the voltage is then simply

$$
\Delta \Phi(y)=i_{0}^{-} \rho_{\|} L
$$

and for all values of $y$ it is controlled by the $c$-axis resistivity $\rho_{\|}$, which vanishes at the glass transition. 
${ }^{1}$ G.W. Crabtree, and D.R. Nelson, Physics Today 50, 38 (1997), and references therein.

${ }^{2}$ G. Blatter et al., Rev. Mod. Phys. 66, 1125 (1994).

${ }^{3}$ G.W. Crabtree, W.K. Kwok, U. Welp, D. López, and J.A. Fendrich, in Proceedings of the NATO Advanced Study Institute on the Physics and Materials Science of Vortex States, Flux Pinning and Dynamics, S. Bose and R. Kossowski, eds. (Kluwer Academic Publishers, 1999).

${ }^{4}$ D.R. Nelson, Phys. Rev. Lett. 60, 1973 (1988).

${ }^{5}$ M.P.A. Fisher, Phys. Rev. Lett. 62, 1415 (1989).

${ }^{6}$ D.S. Fisher, M.P.A. Fisher, and D.A. Huse, Phys. Rev. B 43, 130 (1991).

${ }^{7}$ R.H. Koch et al., Phys. Rev. Lett. 63, 1151 (1989).

${ }^{8}$ P.L. Gammel, L.F. Schneemeyer, and D.J. Bishop, Phys. Rev. Lett. 66, 953 (1991).

${ }^{9}$ D. López, et al., Phys. Rev. Lett. 80, 1070 (1998).

${ }^{10}$ R.C. Budhani, M. Suenaga, H.S. and Liou, Phys. Rev. Lett. 69, 3816 (1992).

${ }^{11}$ M. Konczykowski, et al., Phys. Rev. B 44, 7167 (1991).

${ }^{12}$ L. Civale, et al., Phys. Rev. Lett. 67, 648 (1991).

${ }^{13}$ M. Hawley, I.D. Raistrick, J.G. Beery, and R.J. Houlton, Science 251, 1587 (1991); C. Gerber, D. Anselmetti, J.G. Bednorz, J. Manhort, and D.G. Schlom, Nature 350, 279 (1991).

${ }^{14}$ D.R. Nelson, and V.M. Vinokur, Phys. Rev. Lett. 68, 2398 (1992); Phys. Rev. B 48, 13060 (1993).

${ }^{15}$ G.W. Crabtree, et al., Physica C, 185-189, 282 (1991); W.K. Kwok et al., in Advances in Superconductivity IV, proceedings of the 4th International Symposium on Superconductivity, edited by H. Hayakaswa and N. Koshizuka (Springer, New York, 1992), pp. 317-322.

${ }^{16}$ S.A. Grigera, et al., Phys. Rev. Lett. 81, 2348 (1998). The dominant source of disorder in this experiment was a single family of twin boundaries.

17 D.R. Nelson, and L. Radzihovsky, Phys. Rev. B 54, R6845 (1996).

${ }^{18}$ M.P.A. Fisher, P.B. Weichman, G. Grinstein, and D.S. Fisher, Phys. Rev. B 40, 546 (1989).

19 M. Wallin, and S.M. Girvin, Phys. Rev. B 47, 14642 (1993).

${ }^{20}$ A.J. Lidmar and M. Wallin, Europhys. Lett. 47, 494 (1999).

${ }^{21}$ D.R. Nelson and V.M. Vinokur, to be published.

${ }^{22}$ M.H. Theunissen, E. Van der Drift, and P.H. Kes, Phys. Rev. Lett. 77, 159 (1996).

23 M.C. Marchetti and D.R. Nelson, Phys. Rev. B 59, 13624 (1999).

24 (a) M.C. Marchetti, and D.R. Nelson, Phys. Rev. B 42, 9938 (1990); (b) Physica C 174, 40 (1991).

${ }^{25}$ E. van der Drift, S. Radelaar, A. Pruijmboom, and P.H. Kes, J. Vac. Sci. Technol. B 6, 297 (1988); A. Pruijmboom, P.H. Kes, E. van der Drift, and S. Radelaar, Phys. Rev. Lett. 60, 1430 (1988).

${ }^{26}$ H. Pastoriza, and P.H. Kes, Phys. Rev. Lett. 75, 3525 (1995).

${ }^{27}$ D.R. Nelson, in Phenomenology and Applications of High Temperature Superconductors, edited by K. Bedek et al. (AddisonWesley, New York, 1992), Sec. 4.2.

${ }^{28}$ C.-Y. Mou, R. Wortis, A.T. Dorsey, and D.A. Huse, Phys. Rev. B 51, 6575 (1995).

${ }^{29}$ D.R. Nelson, in Phase Transitions and Critical Phenomena, edited by C. Domb and J.L. Lebowitz (Academic Press, London, 1983), vol.7, and references therein.

${ }^{30}$ D. López, et al., Phys. Rev. B 58, R8913 (1998).

${ }^{31}$ M.C. Marchetti, Phys. Rev. B 43, 8012 (1991).

32 D.A. Huse and S.N. Majumdar, Phys. Rev. Lett. 71, 2473 (1993).

${ }^{33}$ D. López et al., Phys. Rev. Lett. 82, 1277 (1999); G.W. Crabtree, D. Lopez, W.K. Kwok, H. Safar, and L.M. Paulius, in Proceedings of MOS 99, July 28 - Aug. 2, 1999, to be published in J. Low Temp. Phys.

${ }^{34}$ Here $\tau_{0}$ represents the relaxation time of a fluctuation of volume $\sim a_{0}^{2} \xi_{z}$, with $\xi_{z}=\left(\tilde{\epsilon}_{1} / k_{B} T\right) a_{0}^{2}$ the characteristic spacing between entanglements introduced in Ref. [4]. As shown in Ref. [24b], it can be related to the normal state resistivity. It is the time necessary for a segment of size $\xi_{z}$ to diffuse an intervortex spacing, $\tau_{0}=a_{0}^{2} / D$, with $D$ the diffusion constant of a segment of length $\xi_{z}$. This is in turn given by $D=D_{0}\left(c_{0} / \xi_{z}\right)$, with $D_{0}$ the diffusion constant of a vortex in an isolated copper-oxide plane, and $c_{0}$ the average spacing between $C u O_{2}$ planes. As shown in [24b], one finds $\tau_{0} \approx\left(a_{0} \tilde{\epsilon}_{1} / k_{B} T\right)^{2} c^{2} /\left(B H_{c 2} c_{0} \sigma_{n}^{2 d}\right)$, with $\sigma_{n}^{2 d}$ the two-dimensional normal-state conductivity of an isolated $\mathrm{CuO}_{2}$ layer.

${ }^{35}$ M.C. Marchetti, J. Appl. Phys. 69(8), 5185 (1991); L.-W. Chen and M.C. Marchetti, Phys. Rev. B 50, 6382 (1994).

${ }^{36}$ D. López, et. al., Phys. Rev. B 53, R8895 (1996).

${ }^{37}$ In Ref. 32 only the viscosity coefficient $\eta_{z}$ was retained from the outset in the hydrodynamic equations. 